\title{
Single-particle view of stress-promoters induction dynamics: an interplay between MAPK signaling, chromatin and transcription factors
}

\author{
Victoria Wosika and Serge Pelet ${ }^{\star}$ \\ Department of Fundamental Microbiology \\ University of Lausanne \\ 1015 Lausanne, Switzerland.
}

*Correspondance : serge.pelet@unil.ch

\section{Abstract}

Precise regulation of gene expression in response to environmental changes is crucial for cell survival, adaptation and proliferation. In eukaryotic cells, extracellular signal integration is often carried out by Mitogen-Activated Protein Kinases (MAPK). Despite a robust MAPK signaling activity, downstream gene expression can display a great variability between single cells. Using a live mRNA reporter, we monitored the dynamics of transcription in Saccharomyces cerevisiae upon hyper-osmotic shock. The transient activity of the MAPK Hog1 opens a temporal window where stressresponse genes can be activated. Here we show that the first minutes of Hog1 activity are essential to control the activation of a promoter. The chromatin repression on a locus slows down this transition and contributes to the variability in gene expression, while binding of transcription factors increases the level of transcription. However, soon after Hog1 activity peaks, negative regulators promote chromatin closure of the locus and transcription progressively stops. 


\section{Introduction}

2 A crucial function of all cellular life is the ability to sense its surroundings and adapt to

3 its variations. These changes in the extracellular environment will induce specific

4 cellular responses, orchestrated by signal transduction cascades which receive cues

5 from plasma membrane sensors. This information is turned into a biological response

6 by inducing complex transcriptional programs implicating hundreds of genes ${ }^{1-3}$. Tight

7 regulation of signaling is thus crucial to ensure the correct temporal modulation of gene

8 expression, which can otherwise alter the cell physiology ${ }^{4-6}$. Interestingly, single-cell analyses have revealed that genes regulated by an identical signaling activity can display a high variability in their transcriptional responses ${ }^{7-10}$. This noise in transcriptional output questions how signal transduction can faithfully induce different loci and which molecular mechanisms contribute to the variability in gene expression. In eukaryotic cells, various environmental stimuli are transduced by the highly conserved Mitogen-Activated Protein Kinases (MAPK) cascades ${ }^{11,12}$. They control a wide range of cellular responses from cell proliferation, differentiation or apoptosis. In Saccharomyces cerevisiae, a sudden increase in the osmolarity of the medium is sensed by the High Osmolarity Glycerol (HOG) pathway, which leads to the activation of the MAPK Hog1, a homolog of p38 in mammals ${ }^{13,14}$. Upon hyper-osmotic stress, the kinase activity of Hog1 promotes the adaptation of the cells to their new environment by driving an increase in the internal glycerol concentration, thereby allowing to balance the internal and the external osmotic pressures. In parallel to its cytoplasmic activity, Hog1 also transiently accumulates into the nucleus to induce the expression of hundreds of osmostress-responsive genes (Fig. 1a). The MAPK is recruited to promoter regions by Transcription Factors (TFs) and, in turn, Hog1 recruits chromatin remodeling complexes, the Pre-Initiation Complex and the RNA Polymerase II (PollI) to trigger gene expression ${ }^{15,16}$. Once cells have adapted, Hog1 is inactivated and exits the cell nucleus, transcription stops and chromatin is rapidly reassembled at HOG28 induced gene loci.

29 Biochemical analyses of this pathway have identified the key players implicated in gene expression and the central role played by the MAPK in all these steps ${ }^{15}$. In 
parallel, single-cell measurements have uncovered the large variability present in their expression. In particular, translational reporters and RNA-FISH measurements have identified that slow chromatin remodeling promoted by the MAPK at each individual locus is generating strong intrinsic noise in the activation of many stress-responsive genes $^{9,17}$.

In order to get deeper insights into the regulation of osmostress-genes expression kinetics, we aimed at monitoring the dynamics of mRNA production in live single cells. Phage coat protein-based assays, like the MS2 or PP7 systems, have been used to visualize mRNA in live single cells ${ }^{18-20}$. These experiments contributed to revealing the bursty nature of transcription, whereby a set of polymerases simultaneously transcribing a gene generates a burst in mRNA production, which is followed by a pause in transcription ${ }^{21-23}$.

In this study, we dissect the kinetics of transcription of osmostress-genes. The production of mRNA is monitored using the PP7 phage coat protein assay. This reporter allows us to measure with high temporal resolution and in a fully automated manner, the fluctuations in transcription arising in hundreds of live single cells. This analysis enables to dissect the contribution of various players to the overall transcriptional output. We show that the first few minutes of MAPK activity will determine if a gene is transcribed. We also demonstrate that the chromatin state of a promoter will control the timing of activation and thus the variability of the transcription, while the TF binding will influence the level and duration of the mRNA production.

\section{Results}

High osmotic pressure is sensed and transduced in the budding yeast Saccharomyces cerevisiae via the HOG signaling cascade, which culminates in the activation of the MAPK Hog1 (Fig. 1a). Upon activation, this key regulator accumulates in the nucleus to trigger gene expression in a stress level-dependent manner (Supplementary Figure 1a). The activity of the kinase can be monitored by following its own nuclear enrichment ${ }^{24,25}$. In parallel to Hog1, the general stress response pathway is induced by the hyper-osmotic shock and the transcription factor Msn2 also relocate into the nucleus with dynamics highly similar to the ones observed for Hog1 (Supplementary 
Figure $1 \mathrm{~b}$ and c) ${ }^{26,27}$. Nuclear Hog1 and Msn2 (together with its paralog Msn4) induce osmostress-genes expression, with approximately 250 genes being up-regulated upon osmotic shock ${ }^{1,28,29}$. The activity of the pathway is limited to the cellular adaptation time, which conincide with the nuclear exit of Hog1 and the recovery of the cell size (Supplementary Figure 1a and d). The fast and transient activity of the osmostress response as well as the homogenous activation of the MAPK within the population ${ }^{9,25}$ (Supplementary Figure 1e), make this signaling pathway an excellent model to understand the induction of eukaryotic stress-responsive genes, which are often accompanied by important chromatin remodeling.

Monitoring the dynamics of osmostress-genes transcription

In order to quantify the dynamics of transcription in live single cells, we use the PP7 system to label the production of messenger RNAs (mRNA) ${ }^{30}$. Briefly, constitutively expressed and fluorescently labeled PP7 phage coat proteins strongly associate to a binding partner: an array of twenty-four PP7 mRNA stem-loops (PP7sI). In our settings, this PP7 reporter construct is placed under the control of a promoter of interest and integrated in the genome at the GLT1 locus (Fig. 1b) ${ }^{30}$, in a strain bearing a nuclear tag (Hta2-mCherry) and expressing a fluorescently tagged PP7 protein (PP7 $\triangle F G$ GFPenvy ${ }^{31,32}$, abbreviated PP7-GFP, Methods). Upon activation of the promoter, local accumulation of newly synthesized transcripts at the Transcription Site (TS) leads to the formation of a bright fluorescent focus due to the enrichment in PP7-GFP fluorescence above the background signal (Fig. 1c and Supplementary Movie 1). The fluorescence intensity at the TS is proportional to the number of mRNA being transcribed and thus to the instantaneous load of RNA polymerases. After termination, single mRNAs are exported out of the nucleus and their fast diffusion in the cytoplasm prevents their detection under the selected illumination conditions.

Typically, time-lapses with fifteen-second intervals for twenty-five minutes with six Zplanes for the PP7-GFP channel on four fields of view were performed. Image segmentation and quantification were performed automatically, allowing to extract up to four hundred single-cell traces for each experiment ${ }^{33}$. The mean intensity of the 20 brightest pixels in the nucleus, from which the average cell fluorescence was 
91

92

93

94

95

96

97

98

99

100

101

102

103

104

105

106

107

108

109

110

111

112

113

114

115

116

117

118

119

120

subtracted, was used as a measurement of TS intensity and thus as proxy for transcriptional activity (Fig. 1d, Methods).

Fig. 1d displays the average TS fluorescence from more than 200 cells bearing the pSTL1-PP7sI reporter, following the activation of the HOG pathway by various $\mathrm{NaCl}$ concentrations. The HOG-induced STL1 promoter has been extensively studied at the population and single cell leve $\mathrm{e}^{9,17,34,35}$. As expected, increasing salt concentrations lead to a proportionally increasing transcriptional output from the cell population, whereas no change in TS fluorescence is detected in the control medium.

The hundreds of dynamic measurements acquired with the PP7 reporter form a rich dataset where multiple features can be extracted from each single-cell trace (Fig. 1e, Methods). Our automated image segmentation and analysis allow to reliably quantify the appearance (Start Time) and disappearance (End Time) of the TS (Supplementary Figure 2 and Method). The maximum intensity of the trace and the integral under the curve provide estimates of the transcriptional output from each promoter (Fig. 1e). In addition, transcriptional bursts can be identified by monitoring of strong fluctuations in the TS intensity.

\section{Validation of the live $m R N A$ reporter assay}

The mRNA dynamics measured with the PP7 assay are in close agreement with previously reported data set ${ }^{34,36}$. Nonetheless, we also verified with a dynamic protein expression reporter that comparable results can be obtained (Supplementary Figure 3a). The dynamic Protein Synthesis Translocation Reporter (dPSTR) is an assay that allows the kinetics of gene expression from a promoter of interest. It by-passes the slow maturation time of fluorescent proteins (FP) by monitoring the relocation of the fluorescent signal in the nucleus of the cell ${ }^{37}$.

For the PP7 assay, as well as the dPSTR and many other expression reporters, an additional copy of the promoter of interest is inserted in a non-native locus. In order to address if this modified genomic environment alters the dynamics of gene expression, we used CRISPR-Cas9 to integrate the PP7sI downstream of the endogenous STL1 promoter (Supplementary Figure 4). Interestingly, we observe only minor differences between the pSTL1 at its endogenous location and at the GLT1 locus. This observation 
strongly suggests that the STL1 promoter sequence placed at a non-endogenous locus replicates many of the properties of the endogenous promoter.

\section{Intrinsic noise in osmostress-gene activation}

The microscopy images presented in Fig. 1c illustrate the noise that can be observed in the activation of the pSTL1 promoter upon osmotic stress and which has been previously reported ${ }^{9,17}$. In order to verify that this noise is not due to a lack of activation of the MAPK Hog1 in the non-responding cells, we combined the pSTL1-PP7sI reporter and the Hog1-mCherry relocation assay in the same strain. As expected, we observe an absence of correlation between the two measurements (Supplementary Figure 5). Indeed, cells with similar Hog1 relocation behaviors can display highly variable transcriptional outputs.

An additional assay to observe this heterogeneity is to monitor the activation of two STL1 promoters within the same cell. Using a diploid strain where both GLT1 loci were modified with either a pSTL1-24xPP7sI or a pSTL1-24xMS2sI and expressing PP7mCherry and MS2-GFP proteins, we observe an uncorrelated activation of both loci within each single cell (Supplementary Figure 6 and Supplementary Movie 2). This observation confirms the high intrinsic noise generated by the STL1 promoter upon osmotic stress ${ }^{9,37}$. The highly dynamic measurements provided by the PP7 reporter allows us to decipher some of the parameters that contribute to this large variability.

High variability in osmostress-genes transcription dynamics

In addition to PSTL1, five other stress-responsive promoters often used in the literature to report on the HOG pathway transcriptional activity were selected for this study ${ }^{34,38}$. Each reporter strain differs only by the one thousand base pairs of the promoter present in front of the PP7sI (800bp for pSTL1, 660 for pALD39,39); however, each strain displays a different transcriptional response following a $0.2 \mathrm{M} \mathrm{NaCl}$ stimulus (Fig. 2a). Because the level of accumulation of the PP7 signal at the transcription site and the timing of the appearance and disappearance of the TS is different for each tested promoter, it implies that the promoter sequence dictates multiple properties of the transcription dynamics. These dynamic measurements are in general agreement with control experiments performed with the dPSTR assay (Supplementary Figure 3b) and previously published population-averaged data ${ }^{34,37}$. Importantly, expressing three 
times more phage coat proteins did not change the parameters extracted from the PP7 measurements for the two strongest promoters, denoting the absence of titration of PP7-GFP reporter proteins in our experimental settings (Supplementary Figure 7).

The automated analysis allows to identify the presence or absence of a transcription site in each single cell, and thus the fraction of cells that induce the promoter of interest (Fig. 2b). Interestingly, even in absence of stimulus, some promoters display a basal level of transcription. In the pGRE2, pHSP12 and pGPD1 reporter strains, an active transcription site can be detected in 5 to $20 \%$ of the cells in the few time points before the stimulus (Fig. 2c, Supplementary Movie 3). If the period of observation is extended to a twenty-five-minute time lapse without stimulus, this fraction increases 2 to 3-folds (Supplementary Figure 8). Upon activation by $0.2 \mathrm{M} \mathrm{NaCl}$, the fractions of responding cells for the three promoters that display basal expression overcomes $85 \%$, while it remains below $65 \%$ for the three promoters without basal induction.

\section{Chromatin state sets the timing of transcription initiation}

A key parameter controlled by the promoter sequence is the timing of induction. In Fig. $2 \mathrm{~d}$, the time when cells become transcriptionally active (Start Time) is plotted as a Cumulative Distribution Function (CDF) only for the cells where a TS is detected after the stimulus, thereby excluding basal expressing cells and non-responding cells. Treatment with $0.2 \mathrm{M} \mathrm{NaCl}$ results in a sudden activation of transcription (Fig. $2 \mathrm{~d}$ ). This contrasts with non-induced samples, where the CDF of the promoters displaying basal activity rises almost linearly due to stochastic activation during the recording window (Supplementary Figure 8c).

Upon stress, the promoters displaying basal activity are induced faster than the promoters that are repressed under log-phase growth, with pGPD1 being activated the fastest ( 1 min), while $\mathrm{pALD} 3$ and $\mathrm{pSTL} 1$ require more than 4 minutes for activation (Fig. 2e). However, there is a great variability in transcription initiation between cells of the same population, since we generally observe 3 to 4 minutes delay between the $10^{\text {th }}$ and $90^{\text {th }}$ percentiles, with the exception of pGPD1 where the induction is more uniform and less than 2 min delay is observed (Fig. 2e). Comparison between individual replicates demonstrates the reliability of our measurement strategy. Interestingly, we observe a positive correlation between faster transcriptional activation 
183

184

185

186

187

188

189

190

191

192

193

194

195

196

197

198

199

200

201

202

203

204

205

206

207

208

209

210

211

212

213

from $\mathrm{pGPD1}$, pHSP12 and $\mathrm{pGRE2}$ and the presence of basal expression level. These promoters also display the highest numbers of responding cells upon a $0.2 \mathrm{M} \mathrm{NaCl}$ shock. These results suggest that basal expression is associated with a more permissive chromatin state, which enables a faster activation and higher probability of transcription among the cell population.

To test this hypothesis, we disrupted the function of the SAGA chromatin remodeling complex by deleting $G C N 5^{40}$. As expected, we observe fewer transcribing cells and a slower induction of the pSTL1 promoter in this background (Fig. 2f). Less remarkably, abolishing histone $\mathrm{H} 2 \mathrm{AZ}$ variants exchange at +1 and -1 nucleosomes by deleting $H T Z 1^{41}$ only results in a reduced percentage of transcribing cells. Conversely, chromatin state at the STL1 promoter can be loosened by relieving the glucose repression using raffinose as a C-source ${ }^{42}$. Interestingly, a fraction of the cells grown in these conditions displays basal expression from the pSTL1-PP7 reporter and the Start Time in raffinose is accelerated by $1 \mathrm{~min}$ compared to glucose (Fig. $2 \mathrm{~g}$ ).

The link between the chromatin state under log-phase growth and the ability to induce stress-responsive genes is cofirmed by these results. A promoter that is tightly repressed will need more Hog1 activity and thus more time to become transcriptionally active, therefore displaying a lower fraction of responding cells.

\section{Early Hog1 activity dictates transcriptional competence}

The period of Hog1 activity provides a temporal window where transcription can potentially be initiated. However, the switch to a transcriptionally active state takes place almost exclusively within the first few minutes after the stimulus. When comparing the characteristic timing of Hog1 nuclear enrichment to the CDF of Start Times for cells bearing the pSTL1 reporter (Fig. 3a and Supplementary Figure 9a), we observe that $90 \%$ of the transcribing cells initiate transcription during the first few minutes of the stress response, while Hog1 nuclear accumulation rises and before it drops below $80 \%$ of its maximum (decay time). A similar behavior is observed for all the promoters tested, independently of the presence of basal levels (Fig. $3 a$ and b). For $\mathrm{p} A L D 3$, which is the slowest promoter tested, $87 \%$ of the Start Times are detected before the decay of Hog1 activity $(7 \mathrm{~min})$ while the full adaptation time takes $14 \mathrm{~min}$ at $0.2 \mathrm{M} \mathrm{NaCl}$. 
214 Interestingly, promoter output also decreases with the time after stimulus. Cells that start transcribing pSTL1 early display a larger integral over the PP7 signal and a higher maximum intensity compared to cells that initiate transcription later (Fig. $3 \mathrm{c}$ and

217 Supplementary Figure 9b). A similar behavior is quantified for all tested promoters

218 (Supplementary Figures 9c and d). These measurements demonstrate that the high

219 Hog1 activity present in the first minutes of the response is key to determine both the

220 transcriptional state and overall output of the promoters.

TFs control the dynamics and level of mRNA production

Promoters dictate the timing of transcriptional activation of the ORF and the level at which the mRNA is produced. To extract the transcriptional level of each promoter, we use as a proxy the maximum of the PP7 trace of each single cell where a transcription event could be detected (Fig. 4a). This value represents the maximal loading of polymerases on the locus during the period of transcription. Similar results are obtained when comparing the integral below the PP7 trace, which represents the total transcriptional output from a promoter (Supplementary Figure 10a). As shown in Fig. $4 a$, each promoter has an intrinsic capability to induce a given level of transcription, which is independent from the presence of basal transcription or the locus activation time. Indeed, pGRE2 displays the lowest level of induction among all tested promoters,

232 despite the presence of basal transcription and being the second-fastest promoter activated.

As expected, the recruitment of the RNA polymerases is stimulated by the stress; the three promoters with basal activities display a higher transcriptional level upon a $0.2 \mathrm{M}$

$236 \mathrm{NaCl}$ stress than in normal growth conditions (Fig. 4b and Supplementary Figure 10b).

237 Both the general stress transcription factors Msn2 and Msn4 and the TFs activated by 238 the MAPK Hog1 (Hot1, Sko1, Smp1) contribute to the transcriptional up239 regulation $29,38,43$. Based on studies on synthetic promoters, it has been established that 240 binding site number and distance from the Transcription Start Site (TSS) influence the 241 promoter output44. Unfortunately, osmostress promoters display a wide diversity in 242 number and affinity of TF binding sites and no obvious prediction of the transcriptional 243 activity can be drawn (Supplementary Figure 11). While multiple Msn2/4 binding sites 
can be found on the GPD1 and STL1 promoter sequences, their activation are only mildly affected by deletions of these two TFs (Supplementary Figure 12).

Both GPD1 and STL1 are primarily Hog1 targets $28,29,38$. However, their requirements for Hog1 activity is strikingly different. In strains where the MAPK has been anchored

248 to the plasma membrane to limit its nuclear enrichment ${ }^{45}, p S T L 1$ induction is virtually abolished (only $1.5 \%$ transcribing cells) while pGPD1 activity is barely affected (Supplementary Figure 13). Similarly, deletion of either TF Sko1 or Hot1 profoundly alter the capacity of pSTL1 to be induced (Fig. 4c -f) while these same mutations have a weaker effect on the GPD1 promoter.

Because the induction of the STL1 promoter requires an efficient chromatin remodeling, every defect (TF deletion or absence of Hog1 in the nucleus) strongly alters its capability to induce transcription. In comparison, the pGPD1 is less perturbed by these same defects. We postulate that TFs act in a cooperative manner on pSTL1, while they act independently of each other on pGPD1.

\section{Bursts of Polll transcription in osmostress-gene activation}

The PP7 and MS2 systems have allowed to directly visualize transcriptional bursting. In order to identify bursts arising from osmostress promoters, we sought to detect strong fluctuations in each single-cell trace. Fluctuations in TS intensities were filtered to retain only peaks separated by pronounced troughs (Methods). In 20 to $30 \%$ of the traces, two or more peaks are identified (Fig. 5a and b). The total length of the transcript downstream of the promoter is $8 \mathrm{~kb}(1.5 \mathrm{~kb}$ for the stem loops $+6.5 \mathrm{~kb}$ for GLT1). Based on a transcription speed of $20 \mathrm{bp} / \mathrm{s}^{30}$, the expected lifetime of a transcript at the TS is $6.6 \mathrm{~min}$. This corresponds well to the mean duration observed for the pALD3, pCTT1, pSTL1 and pGRE2 reporters (Fig. 5c). However, it is unlikely that the strong TS intensities recorded are generated by a single transcript, but rather by a group of RNA Polll that simultaneously transcribe the locus, probably forming convoys

270 of polymerases ${ }^{46}$. Indeed, single mRNA FISH experiments have shown that following 271 a $0.2 \mathrm{M} \mathrm{NaCl}$ stress, the endogenous STL1 locus produces on average 20 mRNAs per 272 cell, with some cells producing up to $100^{36}$.

273 For $\mathrm{pHSP12}$ and $\mathrm{pGPD1}$, the average peak duration is longer than $11 \mathrm{~min}$ (Fig. 5c), 274 suggesting that multiple convoys of polymerases are traveling consecutively through 
the ORF. Unfortunately, the long half-lives of the transcripts on the locus prevent a separation of individual groups of polymerases. However, when we achieve to isolate

277 individual peaks in the single cell traces, their duration becomes closer to the expected 278 value of $6.6 \mathrm{~min}$ (Fig. $5 \mathrm{~d}$ ). In addition, the output of the transcription estimated by the maximum intensity of the trace or the integral under the whole curve is equal or lower for traces with multiple pulses compared to traces where only a single peak is present (Fig. 5e and Supplementary Figure 14). Together these data strengthen the notion that these stress-responsive promoters are highly processive, displaying an elevated rate of transcription once activated. Only brief pauses in the transcription can be observed in a small fraction of the responding cells.

\section{MAPK activity opens an opportunity window for transcription}

We have shown that transcription initiation is dictated by early Hog1 activity. Next, we want to assess what the determinants of transcription shutoff are and by extension, the duration of transcriptional activity. In the HOG pathway, the duration of transcription has been reported to be limited by the cellular adaptation time ${ }^{34,38}$. Therefore, the duration of transcription is shorter after a $0.1 \mathrm{M} \mathrm{NaCl}$ stress and longer after a $0.3 \mathrm{M}$ stress, compared to a $0.2 \mathrm{M}$ stress (Fig. 6a). For the pSTL1 promoter, the last time point where a PP7 signal is detected at the TS matches the timing of nuclear exit of the MAPK at all concentrations tested (Fig. 6b).

294 In order to challenge this link between Hog1 activity and transcriptional arrest, we sought to modulate the MAPK activity pattern by controlling the cellular environment in a dynamic manner. Using a flow channel set-up, we generated a step, a pulse, or a ramp in $\mathrm{NaCl}$ concentrations (Fig. 6c, Methods). These experiments were performed in a strain carrying the pSTL1-PP7sl reporter in conjunction with Hog1-mCherry, allowing to monitor kinase activity and the downstream transcriptional response in the same cell.

301 The step stimulus at $0.2 \mathrm{M} \mathrm{NaCl}$ mimics the experiments performed in wells, where the concentration of the osmolyte is suddenly increased at time zero and remains constant throughout the experiments (Supplementary Movie 4). The mean responses at the 304 population level (Fig. 6c) confirm this relationship between Hog1 adaptation time and transcription shutoff time. However, at the single cell level, no direct correlation is 
observed between these two measurements due to important single cell variability (Fig. $6 f)$.

In the pulse assay, 7 min after the initial 0.2M step, the $\mathrm{NaCl}$ concentration is set back to OM (Supplementary Movie 5). This shortens the MAPK activity period, as Hog1 leaves the nucleus immediately when cells are brought back in the normal growth medium. Removing the kinase from the nucleus has a direct impact on the transcriptional process. First, fewer cells become transcriptionally active. Second, the active TS sites disappear within a few minutes after the end of the pulse (Fig. $6 \mathrm{~d}$ and e). Therefore in this context, we observe a direct correlation between Hog1 activity and transcription, which is in line with the known role played by MAPKs, and Hog1 in particular, on multiple steps of the transcriptional process ${ }^{47,48}$.

317 The ramp experiment starts with a pulse at $0.2 \mathrm{M} \mathrm{NaCl}$ followed by a slow increase of the $\mathrm{NaCl}$ concentration up to $0.6 \mathrm{M}$ over the next $20 \mathrm{~min}$ (Supplementary Movie 6). This constant rise in external osmolarity extends the Hog1 activity window by preventing the adaptation of the cells. More cells can become transcriptionally active and the transcription shut off is delayed (Fig. $6 \mathrm{~d}$ and e). However, in these conditions, there is a clear lack of correlation between Hog1 activity, which is sustained in many cells over the 30min of the time-lapse, and the transcription output of the pSTL1 that stops much earlier. Taken together, these experiments demonstrate that the MAPK activity is required but not sufficient to sustain the transcriptional process. In the ramp experiment, transcription cannot be sustained throughout the whole Hog1 activity window, demonstrating that other factors contribute to limiting the duration of the transcription.

\section{Promoter identity influences the transcription shutoff time}

In order to test whether the promoter identity plays a role in the process of transcription shutoff, we quantified the duration of the transcriptional period for the six promoters and plotted the cumulative distribution of End Times following a $0.2 \mathrm{M} \mathrm{NaCl}$ stress (Fig. $7 a$ and b). Interestingly, despite similar cell volume adaptation time for all the 334 experiments, the promoters display substantially different kinetics of inactivation. 335 Promoters transcribing at a lower level ( $\mathrm{pCTT1}$ and $\mathrm{pGRE2)}$ terminate transcription earlier. This shorter transcriptional window may reflect an inferior recruitment of 
transcriptional activators to the promoter, enabling an earlier inhibition of transcription due to chromatin closure. In addition, promoters with basal activity display an extended period of transcription after adaptation (Fig. 7a and b). For pGPD1 and pGRE2, this results in a biphasic decay, where the first phase corresponds to the arrest of Hog1induced transcription and the second phase can be associated to the basal transcription arising from these promoters (Fig. 7b). Note that basal transcription may even be increased due to a higher basal Hog1 signaling activity post high osmolarity conditions ${ }^{49}$.

Remarkably, $\mathrm{pHSP} 12$ transcription persists beyond the adaptation time, with nearly $30 \%$ of the cells displaying an active TS at the end of the experiment. This suggests that basal expression from this promoter is strongly increased post-stimulus. In contrast to $\mathrm{pGPD} 1$ and $\mathrm{pGRE2,} \mathrm{pHSP12}$ possesses numerous Msn2/4 binding sites. Although the relocation dynamics of Hog1 and Msn2 are highly similar during the adaptation phase, Msn2 displays some stochastic secondary pulses ${ }^{27}$, that are not correlated to Hog1 relocation events. This could explain the stronger basal expression arising from this promoter post-adaptation (Supplementary Figure 1e and f).

To summarize, these measurements demonstrate that the pattern of MAPK activity provides a temporal window where transcription can take place. When the signaling cascade is shut off, transcription ceases soon afterward. However, the promoter identity, and probably its propensity to recruit positive activators, will determine for how long it can sustain an open chromatin environment favorable to transcription before Hog1 activity decreases due to cellular adaptation.

\section{Discussion}

360 In this study, we have constructed PP7 reporter strains to monitor the transcription 361 dynamics of osmostress promoters. The second exogenous copy of the promoter is 362 integrated at the GLT1 locus. This strategy provides a similar genomic environment for 363 all the promoters, in order to compare their specific characteristics. Interestingly, we saw only minor differences in CDF of Start Times of the pSTL1 when integrated at its endogenous locus compared to the GLT1 locus. This observation provides a strong evidence that TF binding and chromatin state of the duplicated promoter sequences 
mimic closely the ones at the native environment of the gene. Note that the signal at the TS is expected to be proportional to the length of the transcribed mRNA. The GLT1 locus with its $6.5 \mathrm{kB}$ length was expected to provide a signal four times stronger than the endogenous STL1 ORF (1.7kB). The unexpectedly high signal obtained from the PP7 reporter at the endogenous locus may be indicative of global difference in transcription rates between the GLT1 and STL1 ORFs alternatively, the smaller STL1 ORF might enhance transcription efficiency via gene-looping $50,51$.

374 Our data illustrate the complex balance that exists between positive and negative regulators taking place at the stress induced loci. At each locus, positive and negative regulators will control the level and duration of transcription. We have shown that the first few minutes of Hog1 activity are essential to initiate the transcription. Transcription factors, chromatin remodelers such as the SAGA and RSC complexes, together with Hog1 will contribute to open and maintain an accessible chromatin environment at the stress-response loci ${ }^{35,40}$. Once initiated, transcription seems highly processive and only in a small fraction of traces, we are able to detect a pause in transcription. However, it has been shown that Polll recruits additional chromatin remodelers, including the Ino80 complex and Asf1 that will redeposit nucleosomes after acute transcription ${ }^{52}$. These conflicting activities will determine the overall duration of transcription at a locus. Indeed, promoters with lower transcriptional activity, such as pGRE2 and pCTT1, recruit fewer positive activators and will be repressed faster by the negative regulators.

388 The repression level of a promoter during log-phase growth will determine the speed and the noise of the transcription activation process. Thus, for each promoter, a tradeoff has to be found between these two contradictory requirements. For instance, GPD1, which is essential for survival to osmotic stress, has an important basal expression level and can thus be rapidly induced upon stress. Interestingly, the chromatin state, encoded in part by the promoter sequence, can be tuned by external growth conditions. Thus, the noise generated by a promoter is not rigidly set by its DNA sequence but fluctuate based on the environment.

396 In higher eukaryotes, the stress response MAPKs p38 and JNK relocate to the nucleus upon activation ${ }^{53,54}$. Early genes such as c-Fos or c-Jun, are induced within minutes 
after activation of signaling 3,55 . Interestingly, these loci display basal expression and require minimal chromatin modification for their induction ${ }^{56,57}$. Conversely, delayed primary response and secondary response genes require more chromatin remodeling 401 to induce their activation ${ }^{55,58}$. These similarities with Hog1-gene transcriptional regulation suggest a high conservation in the mechanisms used by MAPK in eukaryotes to regulate the dynamics of gene expression.

\section{Methods}

Plasmids and yeast strains

Plasmids and yeast strains used in this study are listed in Supplementary Tables 1 and

2. All strains were constructed in the W303 background. Transformations were performed with standard lithium-acetate protocols. Gene deletions and gene tagging were performed with either pFA6a cassettes ${ }^{59,60}$ or pGT cassettes ${ }^{61}$. Transformants were selected with auxotrophy markers (Uracil, Histidine, Leucine, Tryptophan, Adenine) and gene deletions were performed with antibiotic resistance to Nourseothricin (NAT) or Kanamycin (KAN). In order to generate the membrane anchored Hog1, the pGTT-mCherry vector was modified by inserting annealed oligos encoding the last $30 \mathrm{bp}$ of the Ras2 sequence to obtain the pGTT-mCherry-CaaX plasmid. A strain possessing the Hog1-mCherry:LEU2 modification was transformed with the pGTT-mCherry-CaaX plasmid linearized with Xbal and Sacl to induce a marker switch and introduce the membrane anchoring motif.

\section{PP7 and MS2 strains construction}

419 The PP7-GFP plasmids are based on the bright and photostable GFPenvy fluorescent protein ${ }^{32}$. The PP7 protein was derived from Larson et al. ${ }^{30}$ (Addgene\# 35194) with a truncation in the capsid assembly domain (PP7 $\triangle \mathrm{FG}$ residues 67-75: CSTSVCGE ${ }^{31}$ ). The expression of the PP7 construct is controlled by an $A D H 1$ promoter and a CYC1 terminator. The final construct pVW284 is cloned in a Single Integration Vector URA3

424 (pSIVu $\left.{ }^{61}\right)$. The PP7-mCherry was cloned by replacing the GFP by the mCherry sequence. The MS2-GFP was generated by using the original MS2 sequence from 426 Betrand et al. ${ }^{18}$, which also lacks the capsid assembly domain (Addgene\# 27117), inserted into the pVW284. The PP7 stem-loops plasmids are based on the previously 
published pPOL1 24xPP7sI integrative plasmid30 (Addgene \#35196). The stress responsive promoters replace the POL1 promoter in the original construct using $1 \mathrm{~kb}$ (0.8kb for pSTL1, 0.66kb for pALD3) upstream of the start codon. The pSTL124xMS2sl was generated by replacing the PP7sl with the MS2 stem loops obtained from Betrand et al. ${ }^{18}$ (Addgene\# 31865).

433 A strain bearing the Hta2-mCherry nuclear marker and expressing the PP7-GFP was 434 transformed with plasmids containing the different osmostress promoters driving the 435 PP7sl expression, linearized with a Notl digestion and integrated upstream of the GLT1 ORF, as previously published ${ }^{30}$. Correct integration into the GLT1 locus was screened by colony PCR with primers in the GLT1 ORF (+600 bp) and in the TEF terminator of the selection marker on genomic DNA extractions. The integrity of the PP7 stem-loops array was assessed with primers within the TEF terminator and in GLT1 ORF $(+250$ bp) for all the promoters and deletions, after each transformation performed. For all the strains used in the study, at least two clones with correct genotypes were isolated and tested during a salt challenge time-lapse experiment. From the data analysis, the most frequent phenotype was isolated and the strain selected.

444 To tag the endogenous locus of STL1 with the 24xPP7sl, the plasmid pSP264 with the STL1 promoter was modified by replacing the GLT1 ORF sequence by a 500bp sequence starting $100 \mathrm{bp}$ after the start codon of STL1. The plasmid was digested 447 Sacl-Notl and purified over a gel to isolate a fragment that contains the pSTL124xPP7sl-STL1100-600. A double-strand break was generated in the STL1 ORF using 449 Cas9 and a sgRNA targeting the PAM motif GGG 62 bp upstream of the start codon. 450 The Cas9 and sgRNA are expressed from a $2 \mu$ plasmid (Addgene \# $35464^{62}$ ) slightly 451 modified from the work from Laughery et al. (Addgene\# 6763963). The purified DNA 452 fragment containing the stem-loops was used as repair DNA to promote homologous 453 recombination at the STL1 locus (Supplementary Figure 4a). The correct size of the 454 inserted fragment was verified by colony PCR around the PP7sl insert. Multiple positive clones were screened by microscopy. The results from two transformants are presented in this work to ensure that potentially undesired DNA alterations by Cas9 do not affect the response in the HOG pathway. 
In order to generate the diploid reporter strain, a MATa strain containing the PP7-

mCherry::URA3, pSTL1 24xPP7sl:GLT1 and Hta2-tdiRFP:TRP1 was crossed to a MAT $\alpha$ strain bearing the MS2-GFP::URA3, pSTL1 24xMS2sI:GLT1 and Hta2tdiRFP:NAT. Haploid cells were mixed on a YPD plate for a few hours before cells were resuspended in water and spread with beads on a selection plate (SD-TRP +NAT).

The plasmids generated for this study are available on Addgene.

Yeast culture

Yeast cells were grown in YPD medium (YEP Broth: CCM0405, ForMedium) for transformation or in Synthetic Defined (SD) medium (YNB:CYN3801/CSM : DCS0521, ForMedium) for microscopy experiments. Before time-lapse experiments, cells were grown at least 24 hours in log-phase. A saturated overnight culture in SD medium was diluted into fresh SD-full medium to OD600 0.025 in the morning and grown for roughly 8 hours to reach $\mathrm{OD}_{600}$ 0.3-0.5. In the evening, cultures were diluted by adding $\left(0.5 / \mathrm{OD}_{600}\right) \mu \mathrm{l}$ of cultures in $5 \mathrm{ml}$ SD-full for an overnight growth that kept cells in logphase conditions. Cultures reached an $\mathrm{OD}_{600}$ of $0.1-0.3$ in the morning of the second day and were further diluted when necessary to remain below an $\mathrm{OD}_{600}$ of 0.4 during the day. To prepare the samples, these log-phase cultures were further diluted to an OD 6000.05 and sonicated twice $1 \mathrm{~min}$ (diploids were not sonicated) before placing 200 $\mu l$ of culture into the well of a 96-well glass bottom plate (MGB096-1-2LG, Matrical

478 Bioscience) previously coated with a filtered solution of Concanavalin A diluted to $0.5 \mathrm{mg} / \mathrm{ml}$ in water (C2010, Sigma-Aldrich) ${ }^{64}$. Cells were let to settle for 30-45 minutes before imaging. Osmotic shock was performed under the microscope, by adding 100 $\mu \mathrm{l}$ of a three times concentrated SD-full+NaCl stock solutions to the $200 \mu \mathrm{l}$ of medium already in the well, to reach the final desired salt concentration.

\section{Microscopy}

484 Images were acquired on a fully automated inverted epi-fluorescence microscope (Ti2485 Eclipse, Nikon) placed in an incubation chamber set at $30^{\circ} \mathrm{C}$. Excitation was provided 486 by a solid-state light source (SpectraX, Lumencor) and dedicated filter sets were used 487 to excite and detect the proper fluorescence wavelengths with a sCMOS camera 488 (Flash 4.0, Hamamatsu). A motorized XY-stage was used to acquire multiple fields of 
views in parallel and a piezo Z-stage (Nano-Z200, Mad City Labs) allowed fast Zdimension scanning. Micro-manager was used to control the multidimensional acquisitions $^{65}$.

492 Experiments with PP7sI were acquired with a 60X oil objective. For strains with PP7493 GFP and Hta2-mCherry, GFP (40ms, 3\% LED power) and RFP (20ms), along with two 494 bright field images were recorded every 15 seconds for the GFP and every minute for 495 the other channels, for a total duration of 25 minutes. Six z-stacks were performed on 496 the GFP channels covering $\pm 1.2 \mu \mathrm{m}$ from the central plane with $0.4 \mu \mathrm{m}$ steps. An average bleaching of $32 \%$ for the GFP and $26 \%$ for the RFP for the whole time-lapse was quantified in a strain without the PP7 stem-loops, to avoid artifacts from the appearance of bright fluorescent foci. For all time-lapse experiments, media addition was performed before time point 4 , defined as time zero. All microscopy experiments were performed in duplicate for non-induced control experiment and at least triplicate for the $\mathrm{NaCl}$ induced experiments.

Flow chamber experiments

504 The flow experiments were performed in Ibidi chambers ( $\mu$-Slide VI 0.4, Ibidi). Two $50 \mathrm{ml}$ Falcon tube reservoirs containing SD-full $+0.5 \mu \mathrm{g} / \mathrm{ml}$ fluorescein-dextran (D3305, ThermoFischer) and SD-full $+0.6 \mathrm{M} \mathrm{NaCl}$ were put under a pressure of $30 \mathrm{mbar}$ 507 (FlowEZ, Fluigent). The media coming from each reservoir were connected using FEP tubing (1/16" OD x 0.020" ID, Fluigent) to a 3-way valve (2-switch, Fluigent). The concentration of $\mathrm{NaCl}$ in the medium was controlled using a Pulse-Width Modulation

510 strategy 66,67 . Periods of 4 seconds were used and within this time, the valve controlled 511 the fraction of time when SD-full versus SD-full $+\mathrm{NaCl}$ was flowing. TTL signals generated by an Arduino Uno board and dedicated scripts were used to control 513 precisely the switching of the valve. The fluorescein present in the SD-full medium 514 quantified outside the Cell object provided an estimate of the $\mathrm{NaCl}$ concentration in the medium. Some strong fluctuations in this signal were probably generated by dust particles in the imaging oil or FLSN-dextran aggregates in the flow chamber. Following 517 24hrs log-phase growth, cells bearing the pSTL1-PP7sI reporter, Hog1-mCherry and 518 Hta2-tdiRFP tags were diluted to OD 0.2 , briefly sonicated and loaded in the ibidi 
519 channel previously coated by Concanavalin A. Cells were left to settle in the channel for 10 minutes before SD-full flow was started.

\section{Raffinose experiment}

522 For the experiments comparing pSTL1-PP7sI induction in glucose versus raffinose, 523 cells were grown overnight to saturation in SD-full medium. The cultures were diluted 524 to OD 0.025 (Glucose) or 0.05 (Raffinose) and grown at $30^{\circ} \mathrm{C}$ for at least four hours. 525 In the raffinose medium, the expression level of the PP7-GFP was 2-fold lower than in 526 glucose. Because of this low fluorescence intensity, cells were imaged with a $40 \mathrm{X}$ objective, and a single Z-plane was acquired. Manual curation of the images was performed to define the Start Time in more than 250 cells. This experiment was performed in duplicate.

\section{Data analysis}

531 Time-lapse movies were analyzed in an automated way: cell segmentation, tracking and feature measurements were performed by the YeastQuant platform ${ }^{33}$. Summary of the dataset, strains and cell numbers are provided in Supplementary Table 3. All

534 PP7 experiments were realized in at least two or three fully independent replicate experiments. A representative experiment was selected for each strain and inducing conditions, based on cell size and cell adaptation dynamics. The replicates which did not pass one of these controls were discarded from the replicate analyses. Individual single-cell traces were filtered based on cell shape and GFP intensity to remove segmentation errors or other experimental artifacts. In addition, cells in mitosis were removed from the analysis with a 0.95 filter on the nuclei eccentricity, to remove

541 artifacts from locus and PP7 signal duplication.

542 The Hta2 signal combined with the two bright field images allowed to define the nucleus and cell borders. The GFP z-stacks were converted by a maximum intensity

544 projection in a single image that was used for quantification. In order to avoid improper 545 quantification of transcription sites at the nuclear periphery, the Nucleus object defined by the histone fluorescence was expanded by 5 pixels within the Cell object to define 547 the ExpNucl object. The transcription site intensity was quantified by the difference 548 between the mean intensity of the 20 brightest pixels in the ExpNucl (HiPix) and the median intensity from the whole cell. This provides a continuous trace which is close 
to zero in absence of TS and increases by up to few hundred counts when a TS is present. To identify the presence of a transcription site, a second feature named

552 ConnectedHiPix was used (Supplementary Figure 2a). Starting from the $20 \mathrm{HiPix}$, a 553 morphological opening of the image was performed to remove isolated pixels and 554 retaining only the ones that clustered together which correspond to the transcription site. The ConnectedHiPix value was set to the mean intensity of the pixel present in the largest object remaining after the morphological operation. If no pixel remained after the morphological operation, the ConnectedHiPix was set to NaN. In each singlecell trace, ConnectedHiPix values only detected for a single time point were removed. After this filtering, the first and last time points where a ConnectedHiPix was measured were defined as transcription initiation (Start Time) and shutoff (End Time), respectively. Manual curation of Start and End Times from raw microscopy images was performed to validate this transcription site detection strategy (Supplementary Figure $2 \mathrm{~b}$ and $\mathrm{c}$ ). In order to detect individual transcriptional bursts in the HiPix traces, the findpeak algorithm was used to identify in the trace all the peaks larger than a threshold of 7 counts within the Start and End Times. Following this first process, a set of conditions were defined to retain only the more reliable fluctuations: the drop following the peak has to be larger the fourth of the peak intensity; the intensity of the following peak has to rise by more than a third of the value at the trough. In addition, the value of the peak has to be at least one fifth of the maximum intensity of the trace in order to remove small intensity fluctuations being considered as peaks.

\section{Data Availability}

572 Source data for Figures 1d, 2a, 2f,2g, 3a, 4b, 4c, and 6c and Supplementary Figures $5731,4,5,6,8,12,13$ are provided with the paper. The raw images and additional features 574 measurements that support the findings of this study are available from the 575 corresponding author upon reasonable request.

577 The image analysis platform has been published previously ${ }^{33}$. A more recent version 578 of the code can be obtained from the corresponding author. A script to extract measured parameters from the data is provided as a supplementary file. 


\section{References}

583

584

585

586

587

588

589

590

591

592

593

594

595

596

597

598

599

600

601

602

603

604

605

606

607

608

609

610

611

612

613

614

615

616

617

618

619

620

621

622

623

624

625

626

627

628

1. Gasch, A. P. et al. Genomic expression programs in the response of yeast cells to environmental changes. Mol Biol Cell 11, 4241-4257 (2000).

2. Roberts, C. J. et al. Signaling and circuitry of multiple MAPK pathways revealed by a matrix of global gene expression profiles. Science 287, 873-880 (2000).

3. Ferreiro, I. et al. Whole genome analysis of p38 SAPK-mediated gene expression upon stress. BMC Genomics 11, 144 (2010).

4. Berry, D. B. \& Gasch, A. P. Stress-activated genomic expression changes serve a preparative role for impending stress in yeast. Mol Biol Cell 19, 4580-4587 (2008).

5. Chen, R. E., Patterson, J. C., Goupil, L. S. \& Thorner, J. Dynamic localization of Fus3 mitogen-activated protein kinase is necessary to evoke appropriate responses and avoid cytotoxic effects. Mol Cell Biol 30, 4293-4307 (2010).

6. Formstecher, E. et al. PEA-15 Mediates Cytoplasmic Sequestration of ERK MAP Kinase. Developmental Cell 1, 239-250 (2001).

7. Raser, J. M. \& O'Shea, Erin K. Control of stochasticity in eukaryotic gene expression. Science 304, 1811-1814 (2004).

8. Colman-Lerner, A. et al. Regulated cell-to-cell variation in a cell-fate decision system. Nature 437, 699-706 (2005).

9. Pelet, S. et al. Transient activation of the HOG MAPK pathway regulates bimodal gene expression. Science 332, 732-735 (2011).

10. Corrigan, A. M. \& Chubb, J. R. Regulation of Transcriptional Bursting by a Naturally Oscillating Signal. Current Biology 24, 205-211 (2014).

11. Roux, P. P. \& Blenis, J. ERK and p38 MAPK-Activated Protein Kinases: a Family of Protein Kinases with Diverse Biological Functions. Microbiology and Molecular Biology Reviews 68, 320-344 (2004).

12. Chen, R. E. \& Thorner, J. Function and regulation in MAPK signaling pathways: lessons learned from the yeast Saccharomyces cerevisiae. Biochim Biophys Acta 1773, 13111340 (2007).

13. Saito, H. \& Posas, F. Response to hyperosmotic stress. Genetics 192, 289-318 (2012).

14. Hohmann, S., Krantz, M. \& Nordlander, B. Yeast osmoregulation. Meth Enzymol 428, 2945 (2007).

15. de Nadal, E. \& Posas, F. Multilayered control of gene expression by stress-activated protein kinases. EMBO J 29, 4-13 (2010).

16. de Nadal, E., Ammerer, G. \& Posas, F. Controlling gene expression in response to stress. Nat Rev Genet 12, 833-845 (2011).

17. Neuert, G. et al. Systematic Identification of Signal-Activated Stochastic Gene Regulation. Science 339, 584-587 (2013).

18. Bertrand, E. et al. Localization of ASH1 mRNA particles in living yeast. Mol Cell 2, 437445 (1998).

19. Buxbaum, A. R., Haimovich, G. \& Singer, R. H. In the right place at the right time: visualizing and understanding mRNA localization. Nat Rev Mol Cell Biol 16, 95-109 (2015).

20. Urbanek, M. O., Galka-Marciniak, P., Olejniczak, M. \& Krzyzosiak, W. J. RNA imaging in living cells - methods and applications. RNA Biology 11, 1083-1095 (2014).

21. Lionnet, T. \& Singer, R. H. Transcription goes digital. EMBO Rep 13, 313-321 (2012).

22. Fritzsch, C. et al. Estrogen-dependent control and cell-to-cell variability of transcriptional bursting. Molecular Systems Biology 14, e7678 (2018). 
23. Zoller, B., Little, S. C. \& Gregor, T. Diverse Spatial Expression Patterns Emerge from Unified Kinetics of Transcriptional Bursting. Cell 175, 835-847.e25 (2018).

24. Reiser, V., Ruis, H. \& Ammerer, G. Kinase activity-dependent nuclear export opposes stress-induced nuclear accumulation and retention of Hog1 mitogen-activated protein kinase in the budding yeast Saccharomyces cerevisiae. Mol Biol Cell 10, 1147-1161 (1999).

25. Muzzey, D., Gómez-Uribe, C. A., Mettetal, J. T. \& van Oudenaarden, A. A systems-level analysis of perfect adaptation in yeast osmoregulation. Cell 138, 160-171 (2009).

26. Görner, W. et al. Nuclear localization of the $\mathrm{C} 2 \mathrm{H} 2$ zinc finger protein Msn2p is regulated by stress and protein kinase A activity. Genes Dev. 12, 586-597 (1998).

27. Hao, N. \& O'Shea, E. K. Signal-dependent dynamics of transcription factor translocation controls gene expression. Nature Structural \& Molecular Biology 19, 31-39 (2012).

28. O'Rourke, S. M. \& Herskowitz, I. Unique and Redundant Roles for HOG MAPK Pathway Components as Revealed by Whole-Genome Expression Analysis. Mol Biol Cell 15, 532542 (2003).

29. Capaldi, A. P. et al. Structure and function of a transcriptional network activated by the MAPK Hog1. Nat Genet 40, 1300-1306 (2008).

30. Larson, D. R., Zenklusen, D., Wu, B., Chao, J. A. \& Singer, R. H. Real-Time Observation of Transcription Initiation and Elongation on an Endogenous Yeast Gene. Science 332, 475-478 (2011).

31. Chao, J. A., Patskovsky, Y., Almo, S. C. \& Singer, R. H. Structural basis for the coevolution of a viral RNA-protein complex. Nat. Struct. Mol. Biol. 15, 103-105 (2007).

32. Slubowski, C. J., Funk, A. D., Roesner, J. M., Paulissen, S. M. \& Huang, L. S. Plasmids for C-terminal tagging in Saccharomyces cerevisiae that contain improved GFP proteins, Envy and Ivy. Yeast 32, 379-387 (2015).

33. Pelet, S., Dechant, R., Lee, S. S., van Drogen, F. \& Peter, M. An integrated image analysis platform to quantify signal transduction in single cells. Integrative biology: quantitative biosciences from nano to macro 4, 1274-1282 (2012).

34. de Nadal, E. et al. The MAPK Hog1 recruits Rpd3 histone deacetylase to activate osmoresponsive genes. Nature 427, 370-374 (2004).

35. Mas, G. et al. Recruitment of a chromatin remodelling complex by the Hog1 MAP kinase to stress genes. EMBO J 28, 326-336 (2009).

36. Li, G. \& Neuert, G. Multiplex RNA single molecule FISH of inducible mRNAs in single yeast cells. Sci Data 6, 94 (2019).

37. Aymoz, D., Wosika, V., Durandau, E. \& Pelet, S. Real-time quantification of protein expression at the single-cell level via dynamic protein synthesis translocation reporters. Nature Communications 7, 11304 (2016).

38. Rep, M. et al. Osmotic stress-induced gene expression in Saccharomyces cerevisiae requires Msn1p and the novel nuclear factor Hot1p. Mol Cell Biol 19, 5474-5485 (1999).

39. Alepuz, P. M., de Nadal, E., Zapater, M., Ammerer, G. \& Posas, F. Osmostress-induced transcription by Hot1 depends on a Hog1-mediated recruitment of the RNA Pol II. EMBO J 22, 2433-2442 (2003).

40. Zapater, M., Sohrmann, M., Peter, M., Posas, F. \& de Nadal, E. Selective requirement for SAGA in Hog1-mediated gene expression depending on the severity of the external osmostress conditions. Mol Cell Biol 27, 3900-3910 (2007).

41. Wan, Y. et al. Role of the histone variant H2A.Z/Htz1p in TBP recruitment, chromatin dynamics, and regulated expression of oleate-responsive genes. Mol Cell Biol 29, 23462358 (2009).

42. Ferreira, C. \& Lucas, C. Glucose repression over Saccharomyces cerevisiae glycerol/H+ symporter gene STL1 is overcome by high temperature. FEBS Lett 581, 1923-1927 (2007).

43. de Nadal, E. \& Posas, F. Regulation of gene expression in response to osmostress by the yeast stress-activated protein kinase Hog1. Topics in Current Genetics 20, 81 (2008). 
44. Sharon, E. et al. Inferring gene regulatory logic from high-throughput measurements of thousands of systematically designed promoters. Nat Biotechnol 30, 521-530 (2012).

45. Westfall, P. J., Patterson, J. C., Chen, R. E. \& Thorner, J. Stress resistance and signal fidelity independent of nuclear MAPK function. Proc Natl Acad Sci USA 105, 12212-12217 (2008).

46. Tantale, K. et al. A single-molecule view of transcription reveals convoys of RNA polymerases and multi-scale bursting. Nat Commun 7, 12248 (2016).

47. Pokholok, D. K., Zeitlinger, J., Hannett, N. M., Reynolds, D. B. \& Young, R. A. Activated signal transduction kinases frequently occupy target genes. Science 313, 533-536 (2006).

48. Proft, M. et al. The stress-activated Hog1 kinase is a selective transcriptional elongation factor for genes responding to osmotic stress. Mol Cell 23, 241-250 (2006).

49. Macia, J. et al. Dynamic signaling in the Hog1 MAPK pathway relies on high basal signal transduction. Science Signaling 2, ra13 (2009).

50. O'Sullivan, J. M. et al. Gene loops juxtapose promoters and terminators in yeast. Nat Genet 36, 1014-1018 (2004).

51. Ansari, A. A role for the CPF 3'-end processing machinery in RNAP II-dependent gene looping. Genes \& Development 19, 2969-2978 (2005).

52. Klopf, E. et al. Cooperation between the INO80 complex and histone chaperones determines adaptation of stress gene transcription in the yeast Saccharomyces cerevisiae. Mol Cell Biol 29, 4994-5007 (2009).

53. Cavigelli, M., Dolfi, F., Claret, F. X. \& Karin, M. Induction of c-fos expression through JNKmediated TCF/Elk-1 phosphorylation. The EMBO Journal 14, 5957-5964 (1995).

54. Wood, C. D., Thornton, T. M., Sabio, G., Davis, R. A. \& Rincon, M. Nuclear Localization of p38 MAPK in Response to DNA Damage. Int. J. Biol. Sci. 428-437 (2009) doi:10.7150/ijbs.5.428.

55. Tullai, J. W. et al. Immediate-Early and Delayed Primary Response Genes Are Distinct in Function and Genomic Architecture. J. Biol. Chem. 282, 23981-23995 (2007).

56. Fowler, T., Sen, R. \& Roy, A. L. Regulation of Primary Response Genes. Molecular Cell 44, 348-360 (2011).

57. O'Donnell, A., Odrowaz, Z. A. \& Sharrocks, A. D. Immediate-early gene activation by the MAPK pathways: what do and don't we know? Biochemical Society transactions 40, 5866 (2012).

58. Ramirez-Carrozzi, V. R. et al. Selective and antagonistic functions of SWI/SNF and Mi-2 $\beta$ nucleosome remodeling complexes during an inflammatory response. Genes Dev. 20, 282-296 (2006).

59. Goldstein, A. L. \& McCusker, J. H. Three new dominant drug resistance cassettes for gene disruption in Saccharomyces cerevisiae. Yeast 15, 1541-1553 (1999).

60. Sheff, M. A. \& Thorn, K. S. Optimized cassettes for fluorescent protein tagging in Saccharomyces cerevisiae. Yeast 21, 661-670 (2004).

61. Wosika, V. et al. New families of single integration vectors and gene tagging plasmids for genetic manipulations in budding yeast. Molecular Genetics and Genomics 291, 22312240 (2016).

62. Chee, M. K. \& Haase, S. B. New and Redesigned pRS Plasmid Shuttle Vectors for Genetic Manipulation of Saccharomyces cerevisiae. G3 (Bethesda) 2, 515-526 (2012).

63. Laughery, M. F. et al. New vectors for simple and streamlined CRISPR-Cas9 genome editing in Saccharomyces cerevisiae. Yeast 32, 711-720 (2015).

64. Pelet, S., Aymoz, D. \& Durandau, E. Temporal quantification of MAPK induced expression in single yeast cells. J Vis Exp (2013) doi:10.3791/50637.

65. Edelstein, A., Amodaj, N., Hoover, K., Vale, R. \& Stuurman, N. Computer control of microscopes using $\mu$ Manager. Curr Protoc Mol Biol Chapter 14, Unit14.20 (2010).

66. Unger, M., Lee, S.-S., Peter, M. \& Koeppl, H. Pulse Width Modulation of Liquid Flows: Towards Dynamic Control of Cell Microenvironments. in 15th International Conference on 
miniaturized systems for chemistry and life sciences : Microtas 2011 1567-1569 (Chemical and Biological Microsystems Society, 2011).

67. Unger, M. P. Interrogating the single cell: computational and experimental methods for optimal live cell experiments. (ETH Zurich, 2014). doi:10.3929/ethz-a-010350761.

\section{End Notes}

Acknowledgment

741 We thank members the Pelet lab and Martin lab and for helpful discussions. Marta

742 Schmitt, Yves Dusserre, Gaëlle Spack, Joan Jordan and Clémence Varidel for 743 technical assistance. David Shore and his lab for helpful discussions and reagents. 744 Marie-Pierre Peli-Gulli and Claudio de Virgilio for plasmids, Tineke Lenstra for 745 suggesting the PP7 $\triangle F G$ allele. Agathe Pelet for manual curation of microscopy 746 images. Eulalia de Nadal, Mariona Nadal-Ribelles and Veneta Gerganova for critically 747 reading the manuscript. Work in the Pelet lab is supported by SystemsX.ch (IPhD 748 51PHP0_157354), the Swiss National Science Foundation (SNSF, PP00P3_172900 and 31003A_182431) and the University of Lausanne.

\section{Author Contributions}

VW and SP designed the experiments, analyzed the data and wrote the manuscript. VW established the condition for the PP7 imaging. VW and SP performed the experiments.

Declaration of Interests

The authors declare no competing interests.

\section{Figure Legends}

758 Fig. 1. Monitoring the dynamics of osmostress-genes transcription.

759 a. Schematics of the transcriptional response induced by the MAPK Hog1 upon 760 osmotic stress. Under normal growth conditions, the genomic locus is repressed by 761 histones set in place by the Ino80 complex and Asf1/Rtt109. In addition, H3K4 762 methylated histones mediated by Set 1 contribute to the further repression of the locus 
(upper panel). When Hog1 is active (lower panel), it accumulates in the nucleus with the transcription factors Msn2/4. Hog1 binds to the transcription factors Hot1 and Sko1, allowing the remodeling of the chromatin by Rpd3 and the SAGA complex. The polymerases can be recruited to the locus and the RSC and SWR complexes evict nucleosomes on the ORF. b. Construction of the transcriptional reporter. The promoter of interest ( $\mathrm{pPROM}$ ) is cloned in front of 24 stem-loops (24xPP7s). This construct is transformed in yeast and integrated in the GLT1 locus 5'UTR, replacing the endogenous promoter. Upon induction of the promoter, the mRNA stem-loops are transcribed and recognized by the fluorescently-tagged PP7 phage coat protein. $\mathbf{c}$. Maximum intensity projections of Z-stacks of microscopy images from the pSTL1PP7sl reporter system in a $0.2 \mathrm{M} \mathrm{NaCl}$ osmotic stress time-lapse experiment. The appearance of bright foci (arrow heads) in the nucleus of the cells denotes the active transcription arising from the promoter. Scale bar represents $5 \mu \mathrm{m}$. d. Dynamics of the pSTL1-PP7sI transcription site intensity (20 brightest pixels in the nucleus minus the median fluorescence of the cell) following hyperosmotic stress. The mean from 200 to 400 cells is represented by the solid line. The shaded areas represent the s.e.m. e. Analysis of one representative single-cell trace. The raw trace (gray) is smoothed with a moving average (dark blue) and normalized by subtracting the intensity of the first time point after the stimulus. Multiple quantitative values can be extracted from this trace (see Methods). Source data are provided for $\mathrm{d}$.

Fig. 2. Chromatin state dictates the transcription initiation of stress-induced promoters

a. Dynamics of the transcription site intensity from six different promoters following a $0.2 \mathrm{M} \mathrm{NaCl}$ stress. The mean of at least 140 cells is represented by the solid line. The shaded areas represent the s.e.m. b. Percentage of cells where a PP7 TS site was detected. The light shaded area represents the percentage of PP7 positive cells before the stimulus was added (basal transcription).

c. The microscopy thumbnails display cells bearing the pGPD1-PP7sI reporter system, where transcription sites (arrow

792 heads) can be detected before and after the stress of $0.2 \mathrm{M} \mathrm{NaCl}$. Scale bar represents 
considering only the cells that induce transcription after time zero. e. $10^{\text {th }}, 50^{\text {th }}$ and $90^{\text {th }}$ percentiles of the Start Times shown for the two to three replicates measured for each promoter. The number of stars next to each measurement corresponds to the number of promoters without basal level that are significantly different from the promoter with basal level (two-sample $t$-test, $\mathrm{p}<0.05$ ). f. Cumulative distribution functions of Start Times for the pSTL1-PP7sl strain in wild type, $h t z 1 \Delta$ or gcn5 $\Delta$ backgrounds. The inset shows the percentage of PP7 positive cells in each background. g. Cumulative distribution functions of Start Times for the pSTL1-PP7sI strain grown in glucose or raffinose. The inset shows the percentage of PP7 positive cells, the light blue bar the basal positive PP7 cells. Source data are provided for a, f, $g$.

Fig. 3. Early Hog1 activity dictates promoter activation and output

a. In Hog1 nuclear relocation traces obtained from single cells, the timing of Hog1 nuclear entry $(\bullet)$, maximum enrichment $(\bullet)$, start of the decay in nuclear enrichment and $25^{\text {th }}$ to $75^{\text {th }}$ percentiles (lines) for these measurements are plotted for three different osmotic stresses (central panel). The cumulative distribution functions of Start

811 Times for the pSTL1-PP7sI reporter for these same three concentrations are plotted

812 (lower panel). b. Histograms of Start Times following a 0.2M stress for the five other 813 promoters tested. The vertical dashed line represents the median decay time of Hog1 measured at $0.2 \mathrm{M}$. The number in the legend indicates the percentage of cells which have initiated transcription before the median Hog1 decay time. c. The population of pSTL1-PP7sI positive cells is split in four quartiles based on their Start Time. The

817 median $(\bullet)$ and $25^{\text {th }}$ to $75^{\text {th }}$ percentiles (line) of the integral of the PP7 trace is plotted for each quartile. Source data are provided for a.

Fig. 4. Transcription factors control the dynamics and level of mRNA production

821 a. Violin plots of the trace intensity (maximum of the TS during the transcription period) 822 for the six promoters after stimulation by $0.2 \mathrm{M} \mathrm{NaCl}$. Each dot represents the value 823 calculated from a single cell. The solid line is the median and the dashed line the mean 
of the population. b. Comparison between the trace intensity in stimulated $(0.2 \mathrm{M} \mathrm{NaCl})$ and unstimulated conditions $(0.0 \mathrm{M})$ for the three promoters displaying basal expression. c. - f. Effect of the deletions of the HOT1 and SKO1 transcription factor genes on PSTL1 and PGPD1 dynamics of transcription (c), cumulative distribution functions of Start Times (d), the trace intensity (e) and the percentage of responding cells (f) for the pSTL1-PP7sI and pGPD1-PP7sI reporter strains following a $0.2 \mathrm{M} \mathrm{NaCl}$ stress for at least 200 cells. For the pSTL1-PP7sI hot1 $\Delta$ sample, 349 cells were analyzed with only 9 displaying a PP7 positive signal. This low number does not allow to draw a meaningful CDF curve in panel $d$. Source data are provided for $b$ and $c$.

Fig. 5. Identification of transcription bursts in stress-induced transcription.

a. Percentage of cells where 1,2 and 3 or more peaks are identified among the population of responding cells for the different promoters following a $0.2 \mathrm{M} \mathrm{NaCl}$ stress.

b. Examples of single-cell traces displaying 1 or 2 peaks for the pSTL1-PP7sI and the pGPD1-PP7sI reporter strains. c. Violin plots representing the Peak Duration. Each dot represents the value calculated for a single peak. The solid line is the median and the dashed line the mean of all the peaks measured. $\mathbf{d}$. - e. The population of cells was split between cells displaying one peak and two or more peaks. The Peak Duration (d) and Trace Intensity (e) are plotted for the pHSP12-PP7sI and pGPD1-PP7sI strains. Each dot represents the value calculated for a single peak (d) or a single cell (e). The solid line is the median and the dashed line the mean of the population.

Fig. 6. Hog1 activity and promoter identity control the shutoff of transcription

847 a. Violin plots representing the Transcription Period (time difference between End Time and Start Time) measured for the pSTL1-PP7sl reporter following 0.1, 0.2 and $0.3 \mathrm{M}$ $\mathrm{NaCl}$ stresses. Each dot represents the value calculated from a single cell. The solid line is the median and the dashed line the mean of the population. b. One minus the cumulative distribution function of End Times for the pSTL1-PP7sl reporter. The vertical dashed lines represent the median adaptation time of Hog1 for the three different stress levels. c. Dynamics of the estimated $\mathrm{NaCl}$ concentrations in the 854 medium for the pulse, step and ramp experiment protocols (upper panel, Methods). 
855 Corresponding Hog1 relocation dynamics (middle panel) and pSTL1-PP7sl 856 transcription site intensity (lower panel). The mean of at least 180 cells is represented 857 by the solid line. The shaded areas represent the s.e.m. d. Cumulative distribution 858 function (CDF) of the Start Time for all cells in the pulse, step and ramp experiments. 859 The CDF at 15 min represents the fraction of responding cells for each condition. e. 860 One minus the cumulative distribution function of End Times only for the responding 861 cells in the pulse, step and ramp experiments. f. Correlation between the Hog1 862 adaptation time and the PP7 End Time measured in the same cells in the pulse, step 863 and ramp experiments. The open markers indicate cells where Hog1 has not adapted 864 at the end of the time lapse. Adaptation time is arbitrarily set to $35 \mathrm{~min}$ for this sub865 population. Source data are provided for $\mathrm{C}$.

866

867 Fig. 7. Transcription shutoff from different promoters

868 a. Violin plots representing the Transcription Period measured for the six different 869 promoters following a $0.2 \mathrm{NaCl}$ stress. Each dot represents the value calculated from 870 a single cell. The solid line is the median and the dashed line the mean of the 871 population. b. One minus the cumulative distribution function of End Times for the 872 different promoters. The vertical dashed line represents the median adaptation time of $873 \mathrm{Hog} 1$ at $0.2 \mathrm{M} \mathrm{NaCl}$. 
bioRxiv preprint doi: https://doi.org/10.1101/728329; this version $\$$ sted February 3, 2020. The copyright holder for this preprint (which a was not certified by peer review) is the author/funder, who has graded bioRxiv a license to display the preprint in perpetuity. It is made
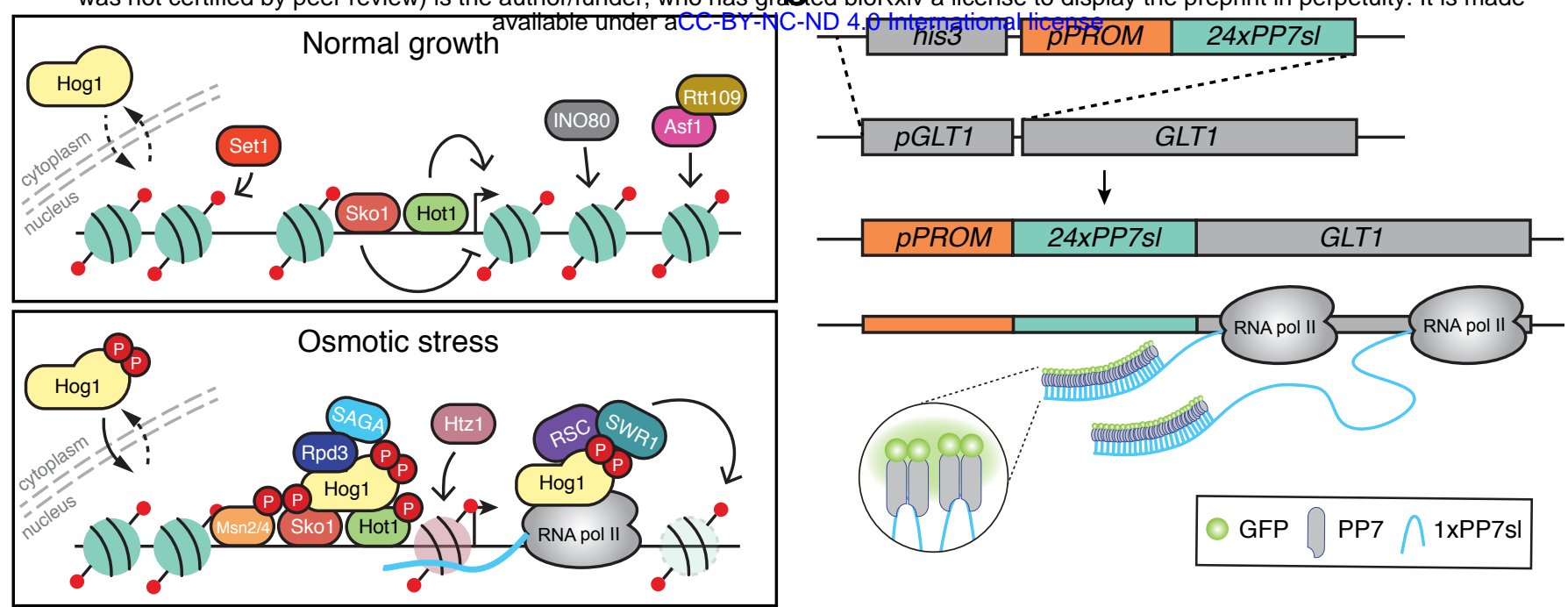

1) nucleosome I) evicted nucleosome I) H2AZ containing nucleosome

i H3K4me $P$ phosphorylation $\rightarrow$ positive regulation $\rightarrow$ negative regulation

C
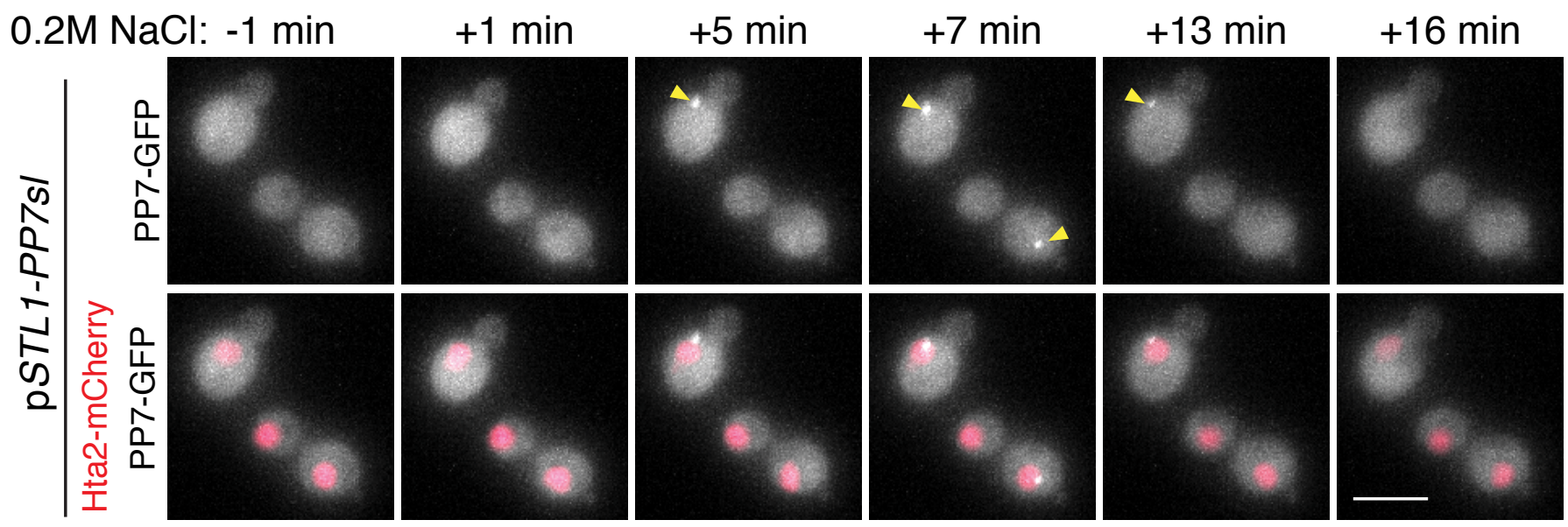

d

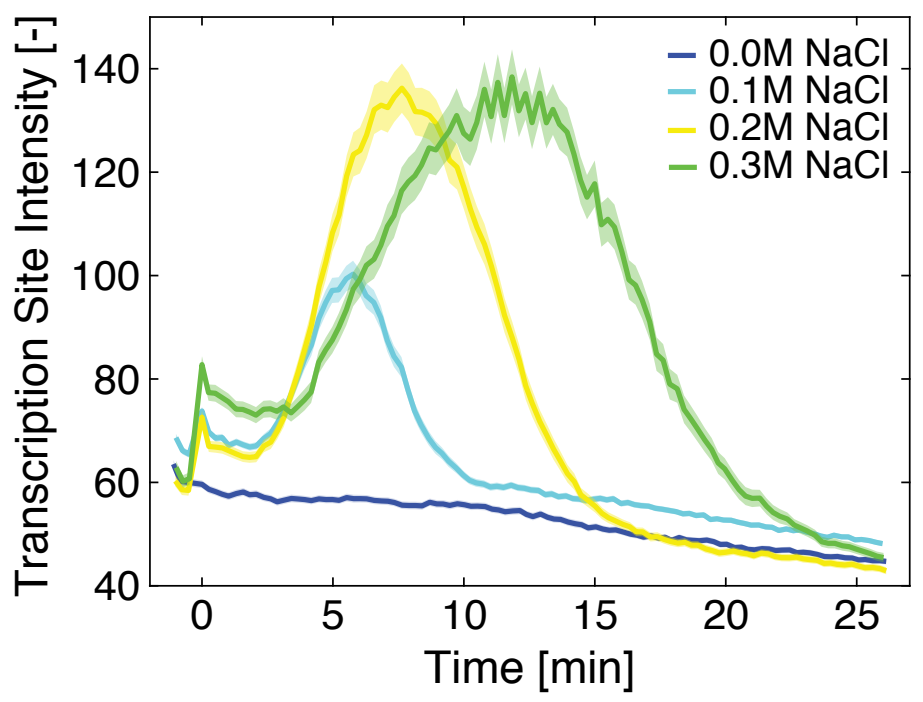

e

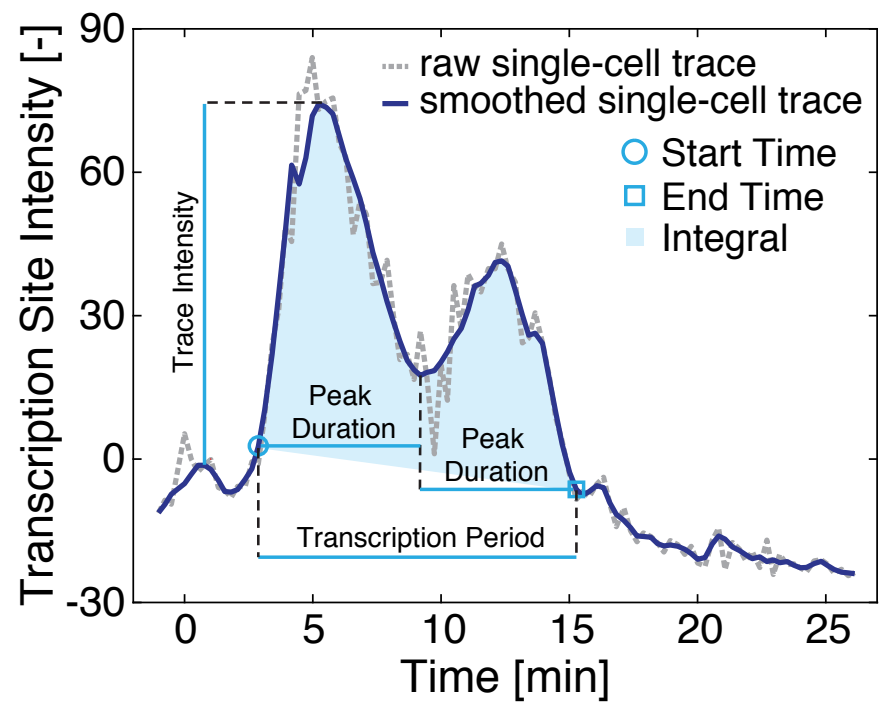


bioRxiv preprint doi: https://doi.org/10.1101/728329; this version posted February 3, 2020. The copyright holder for this preprint (which

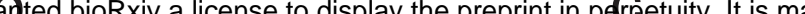
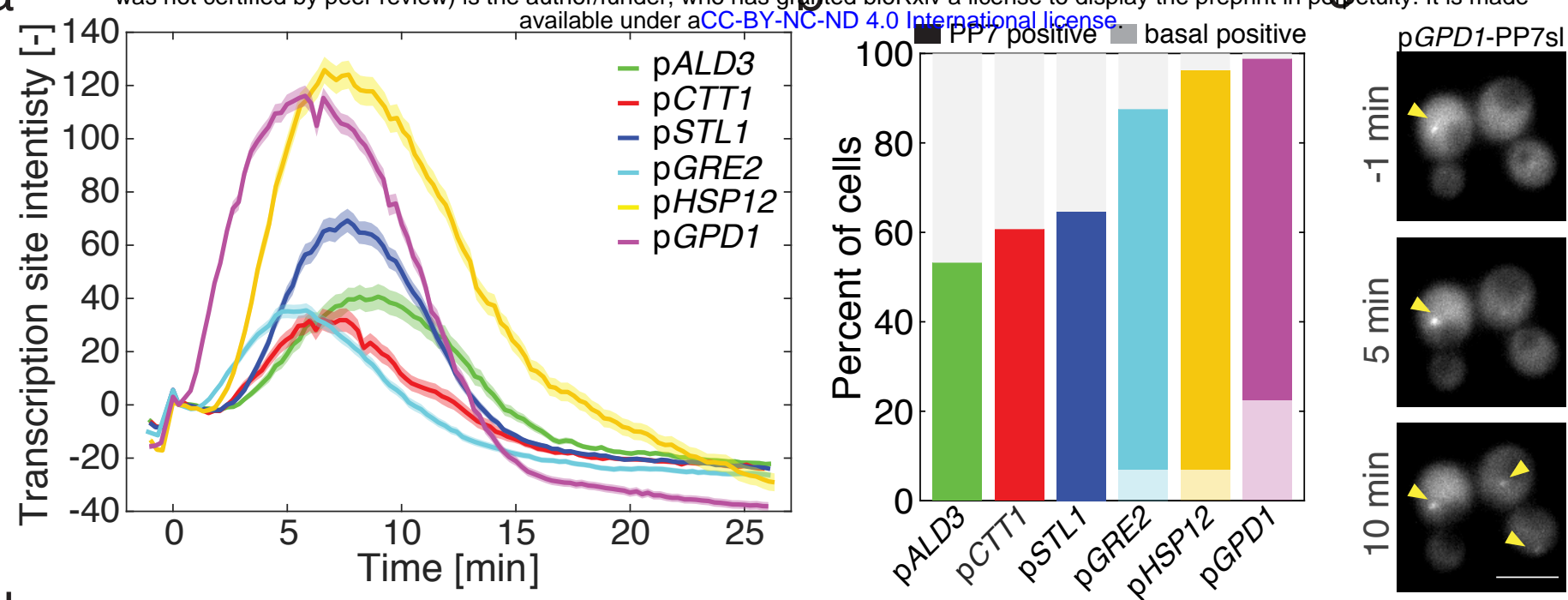

d

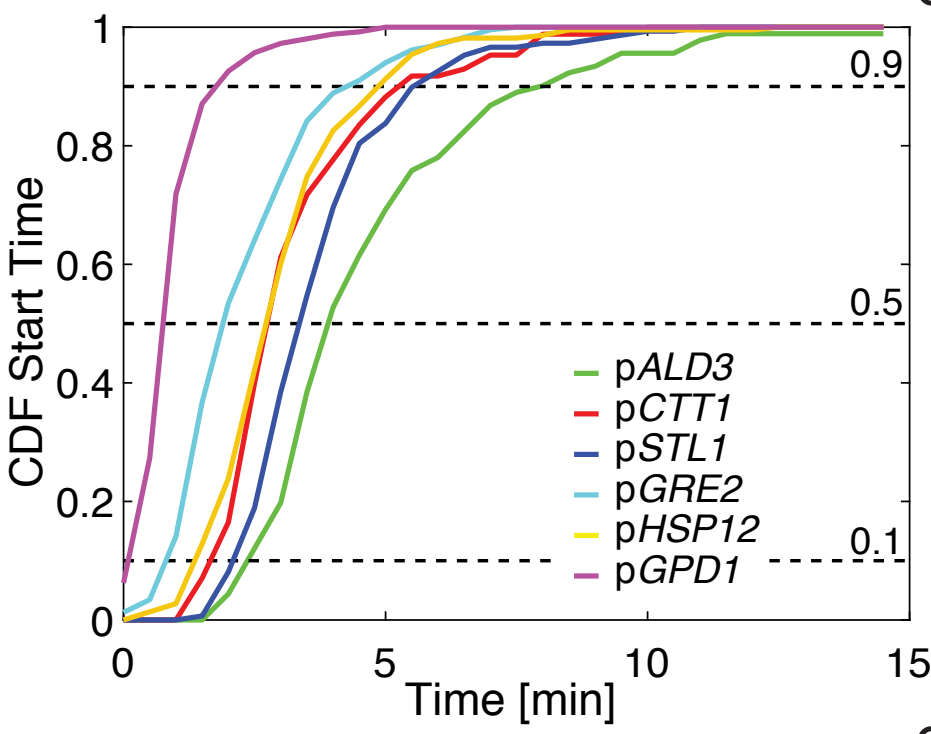

f

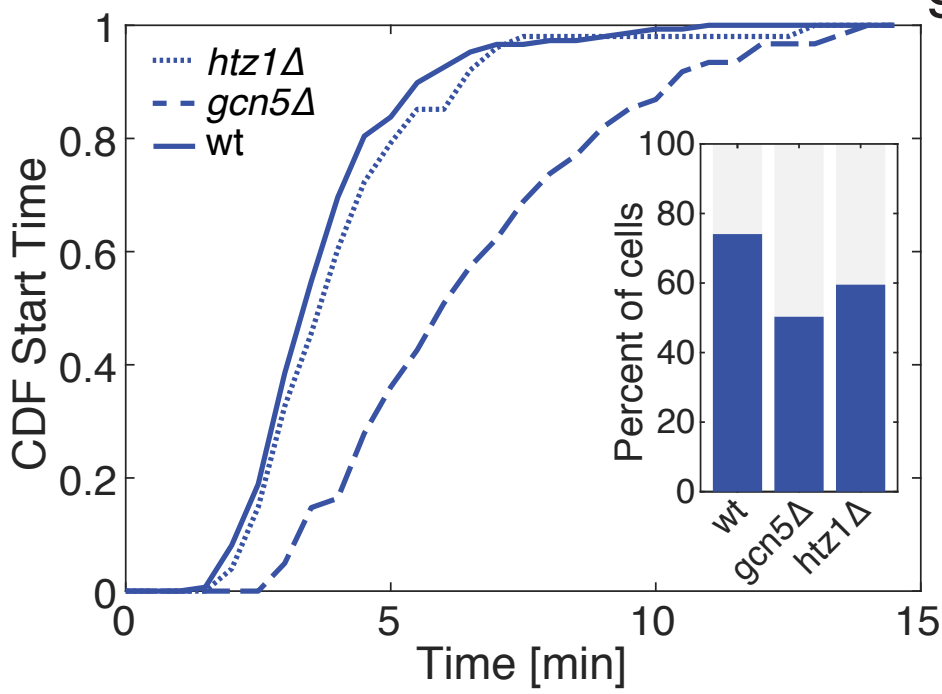

e

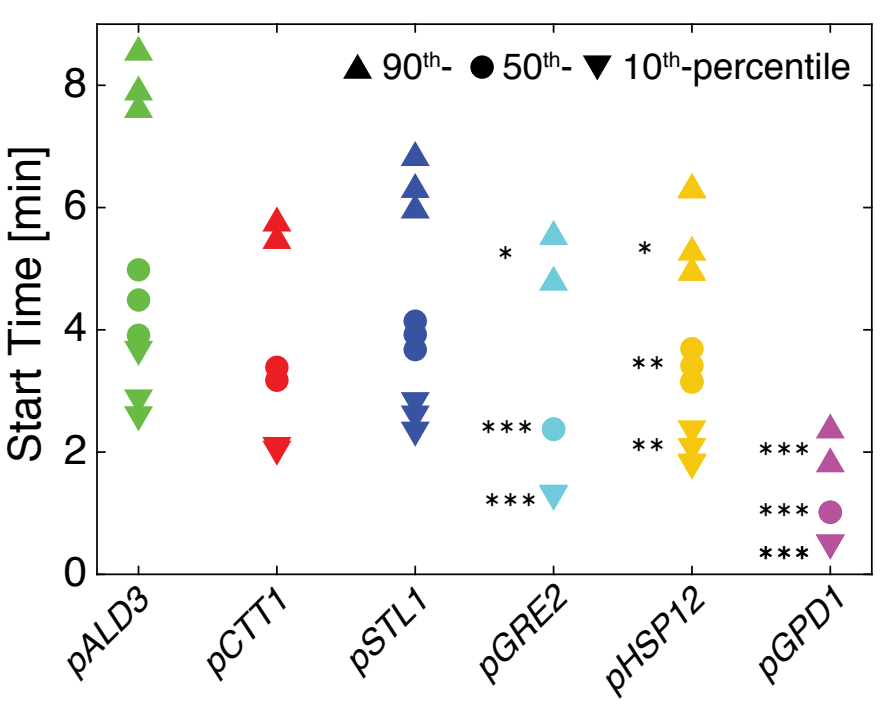

9

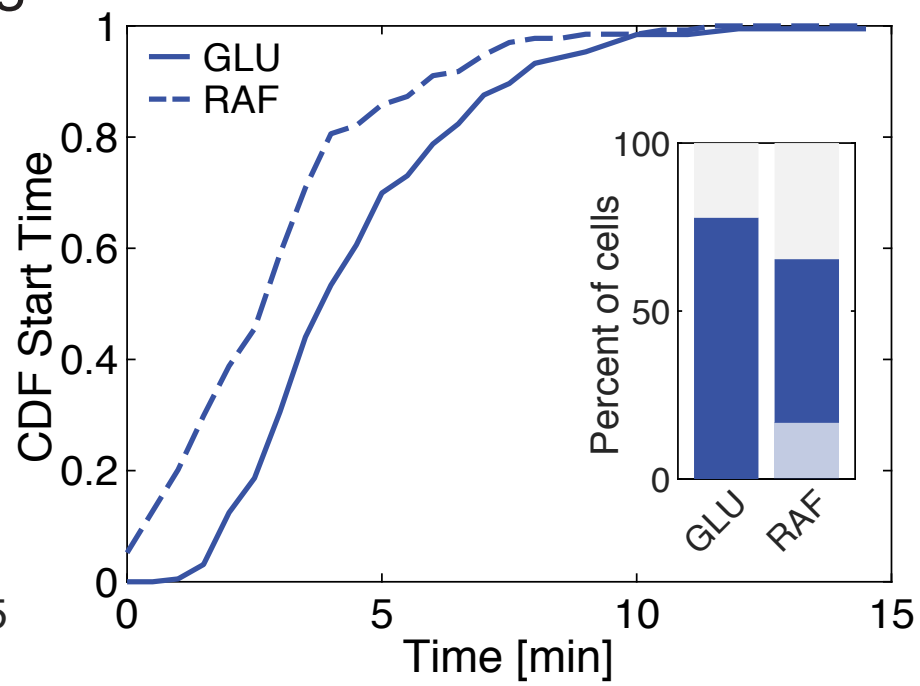


bioRxiv preprint doi: https://doi.org/10.1101/728329; this version posted February 3, 2020. The copyright holder for this preprint (which was not certified by peer review) is the author/funder, who has granted bioRxiv a license to display the preprint in perpetuity. It is made

a available under aCC-BY-NC-ND 4.0 International license.
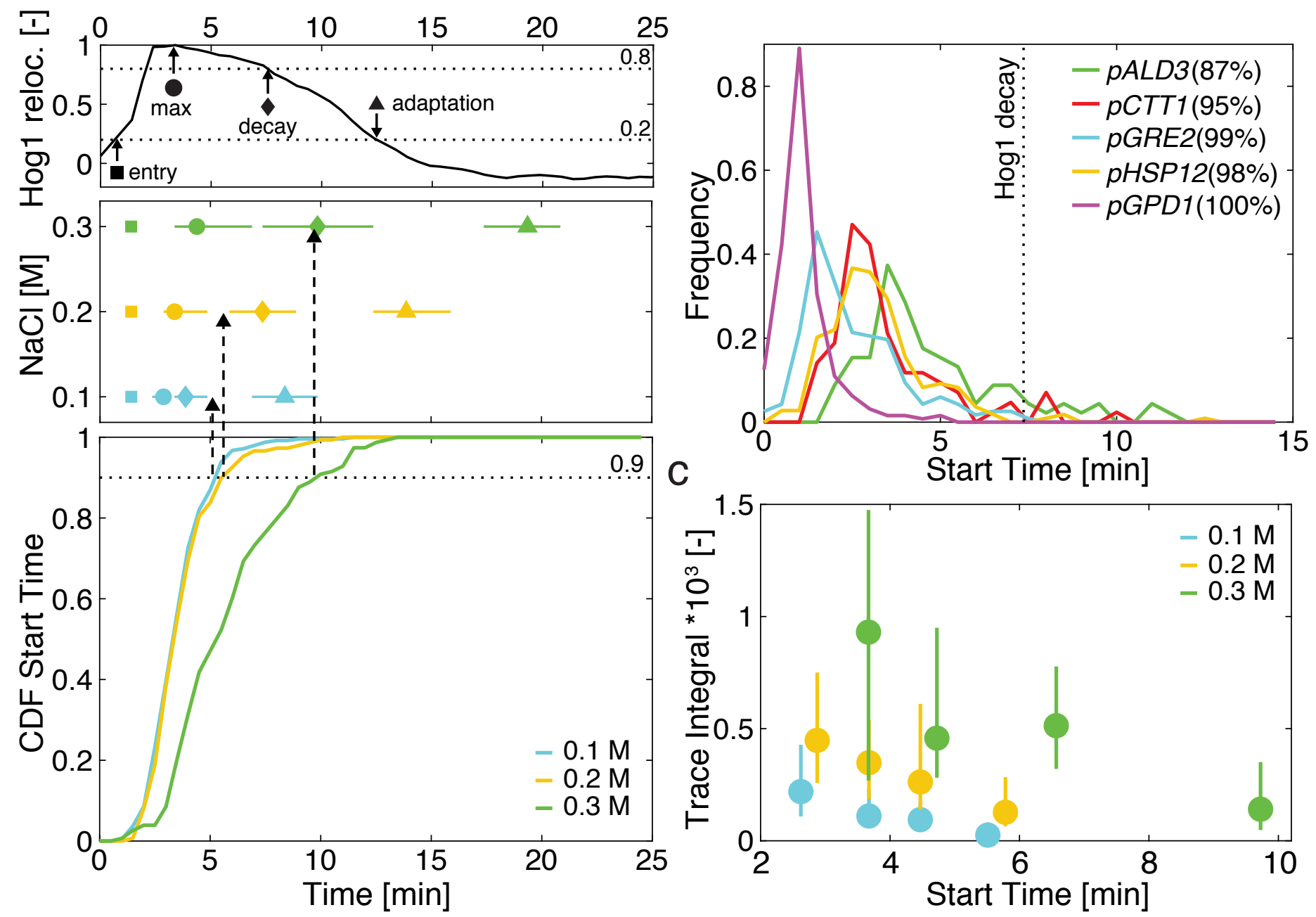

available under aCC-BY-NOQD 4.0 International license.

C
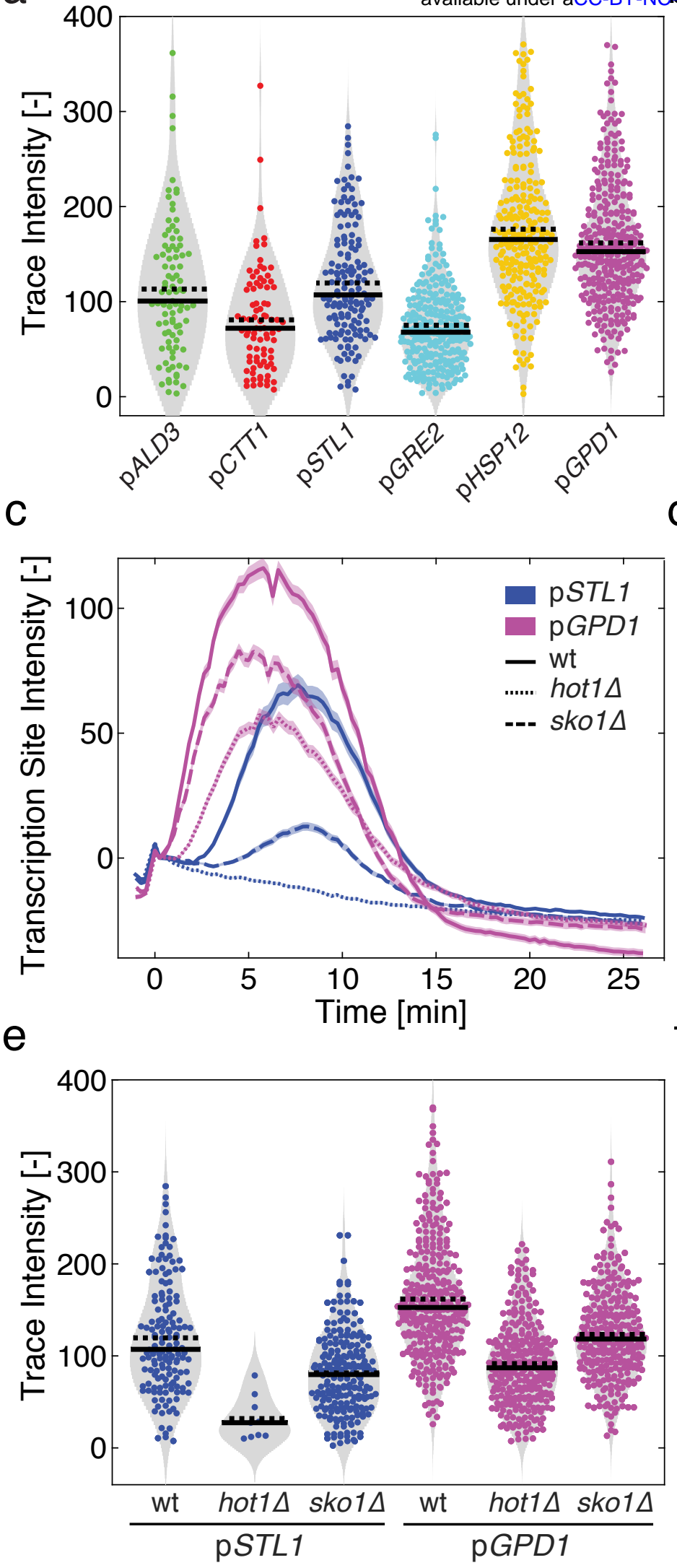

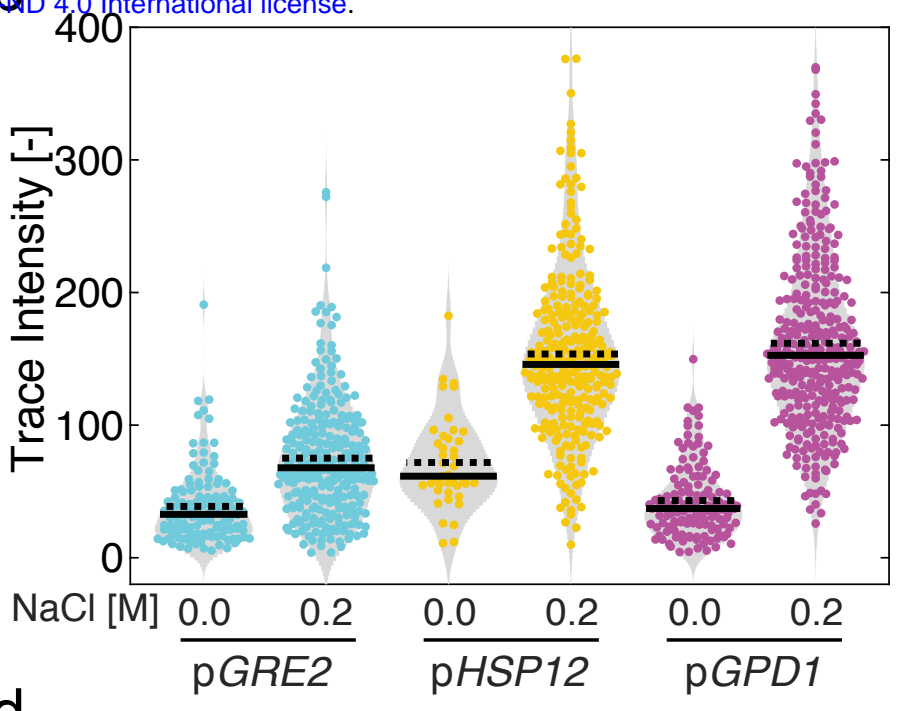

d
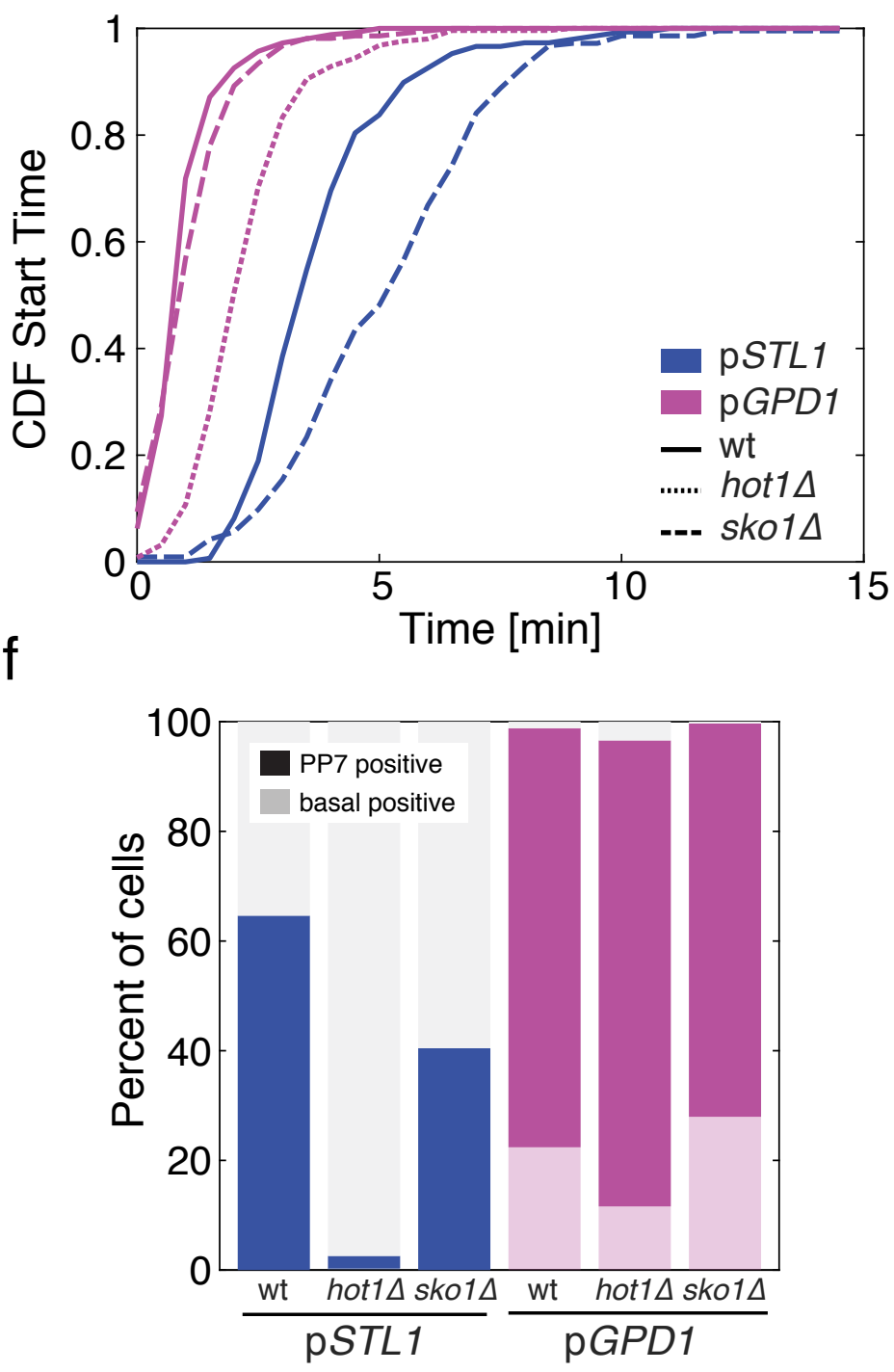
bioRxiv preprint doi: https://doi.org/10.1101/728329; this version posted February 3, 2020. The copyright holder for this preprint (which was not certified by peer review) is the author/funder, who has granded bioRxiv a license to display the preprint in perpetuity. It is made
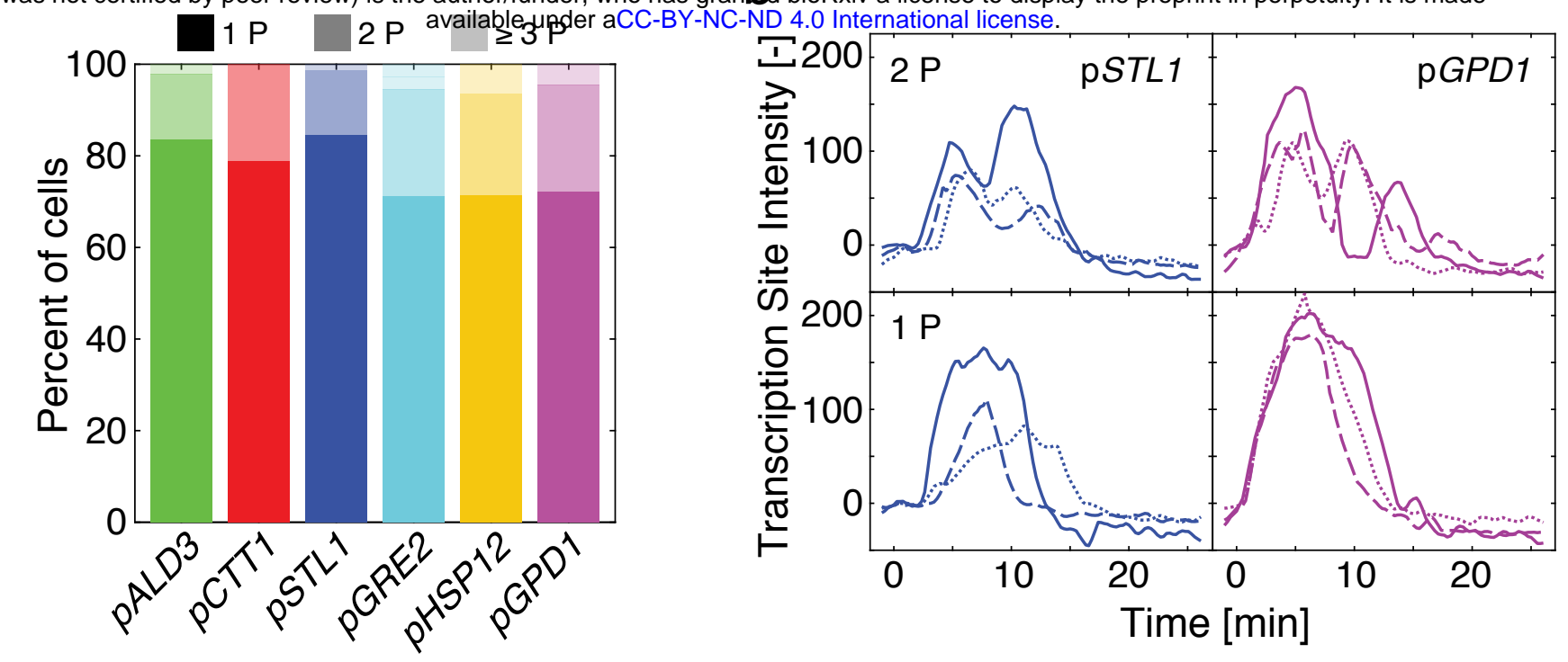

C

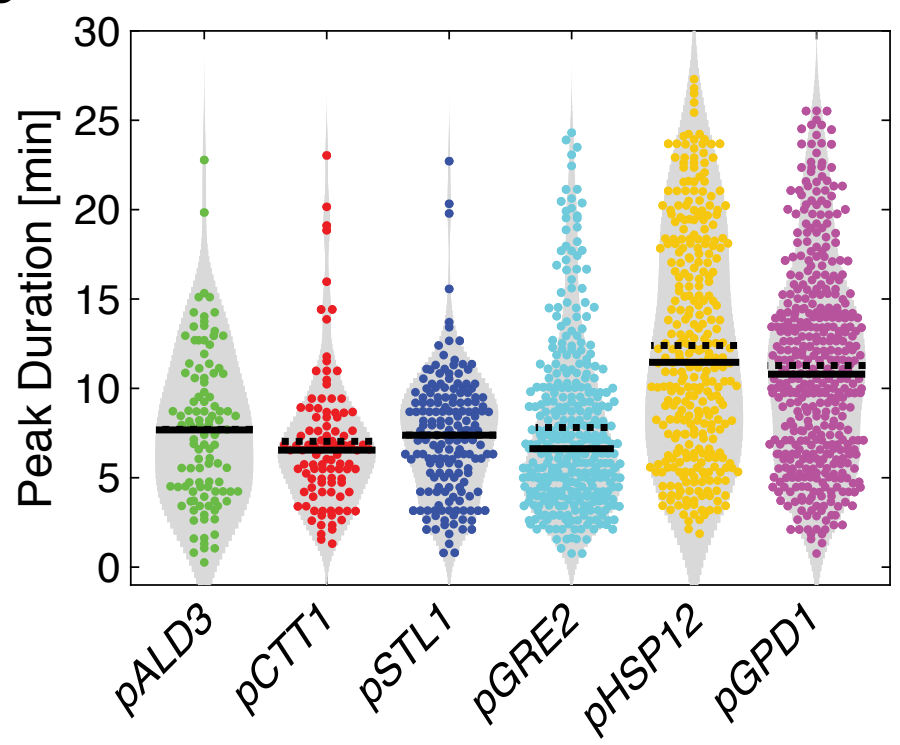

d

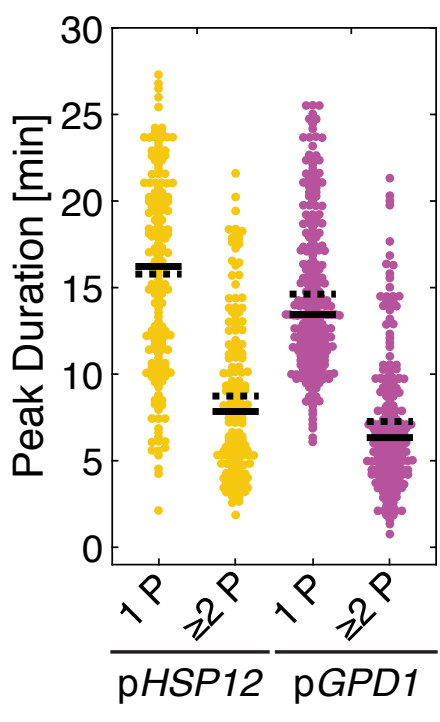

e

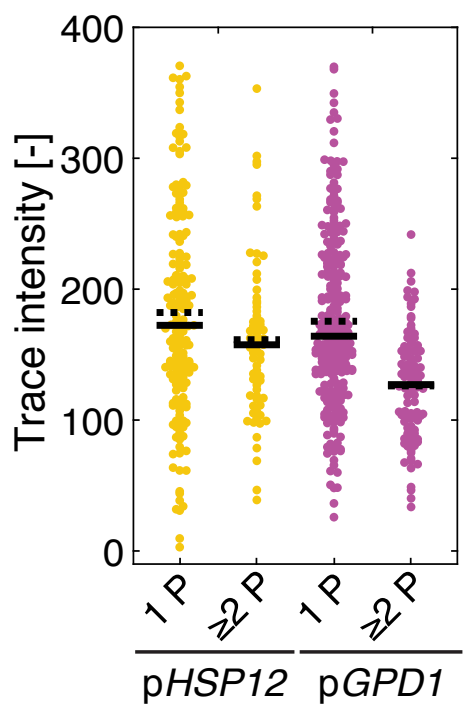


bioRxiv preprint doi: https://doi.org/10.1101/728329; this version posted February 3, 2020. The copyright holder for this preprint (which was not certified by peer review) is the author/funder, who has granted bioRxiv a license to display the preprint in perpetuity. It is made

a

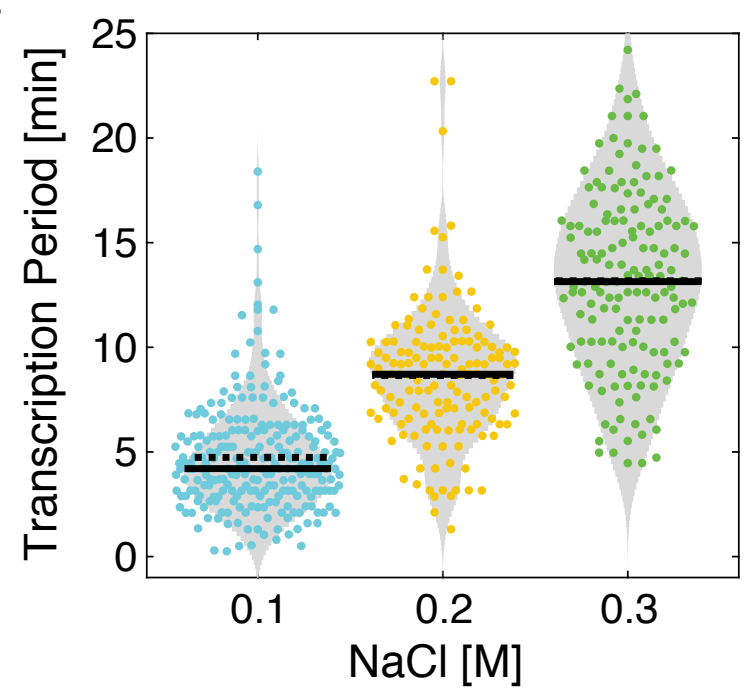

C

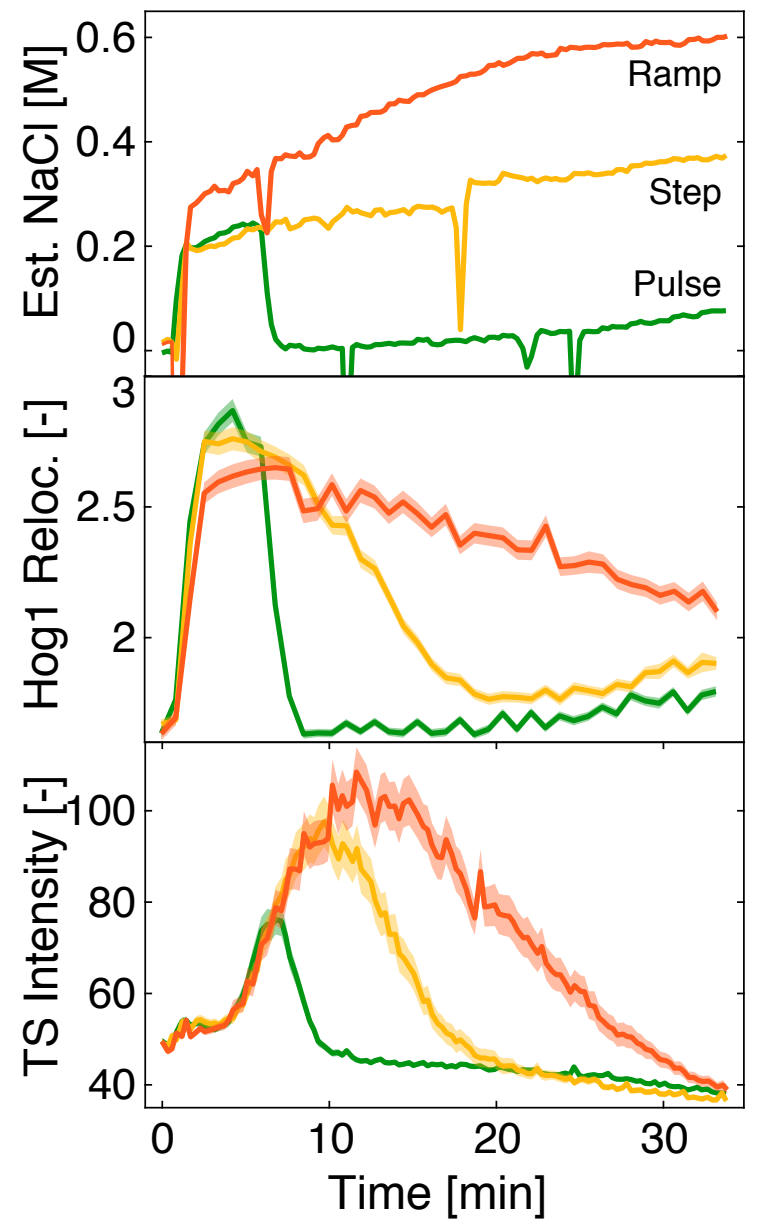

NG-ND 4.0 International license.
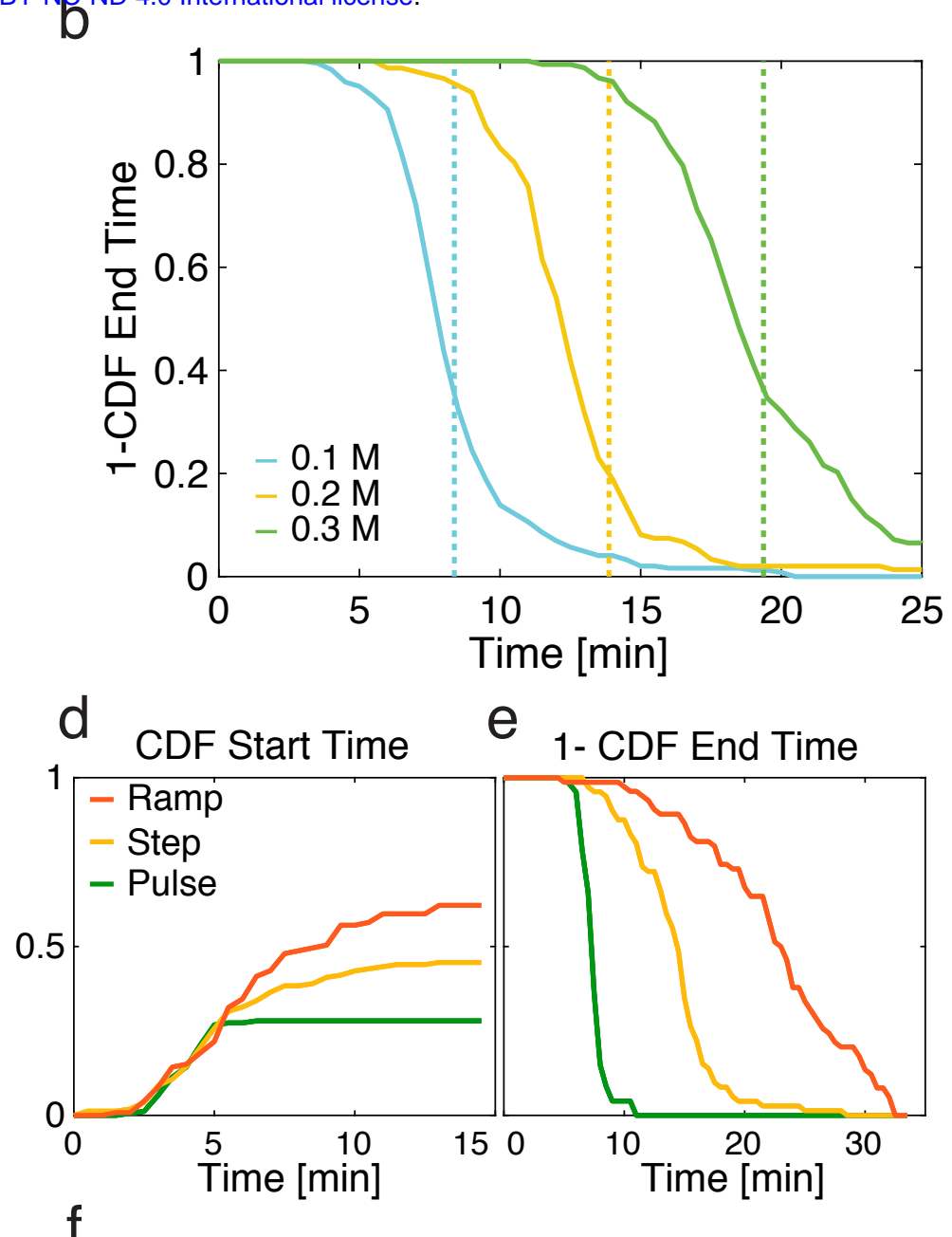

f

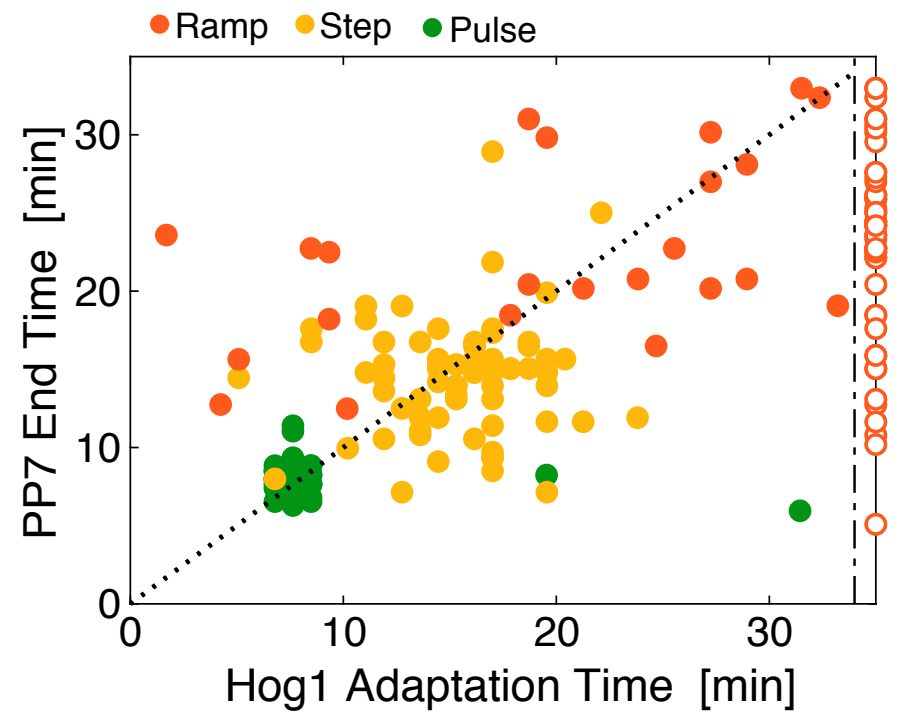


a

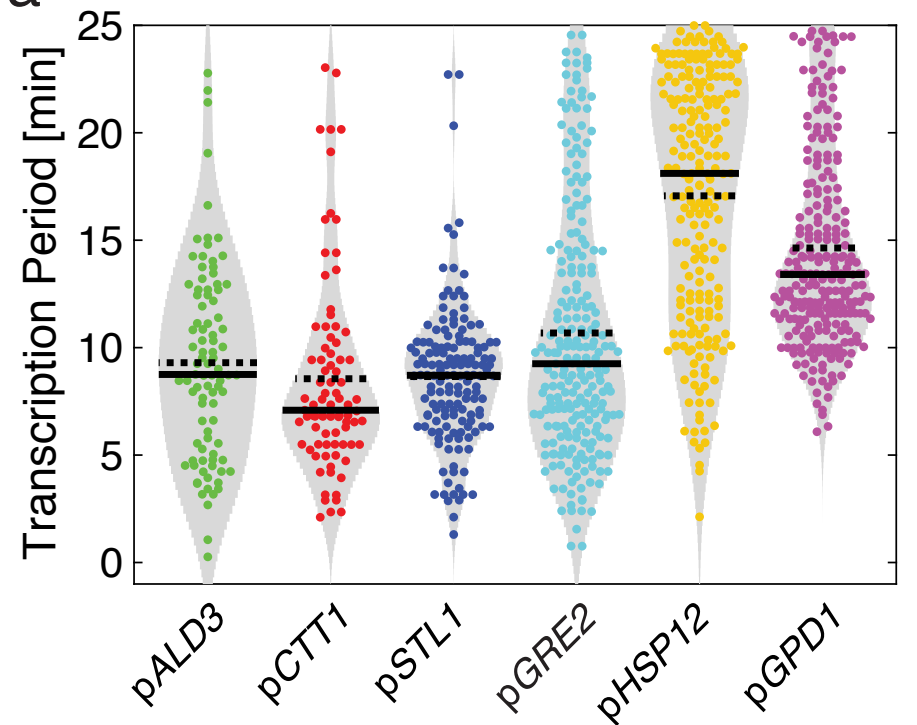

b

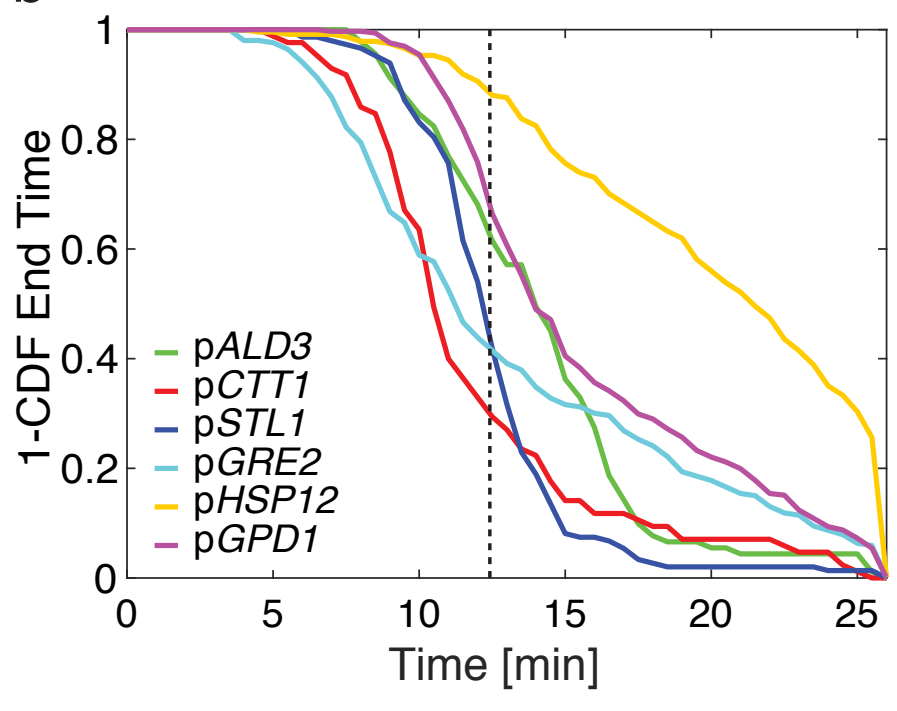

